\title{
Evaluation of a human neurite growth assay as specific screen for developmental neurotoxicants
}

\author{
Anne K. Krug • Nina V. Balmer • Florian Matt • \\ Felix Schönenberger $\cdot$ Dorit Merhof $\cdot$ Marcel Leist
}

\begin{abstract}
Organ-specific in vitro toxicity assays are often highly sensitive, but they lack specificity. We evaluated here examples of assay features that can affect test specificity, and some general procedures are suggested on how positive hits in complex biological assays may be defined. Differentiating human LUHMES cells were used as potential model for developmental neurotoxicity testing. Forty candidate toxicants were screened, and several hits were obtained and confirmed. Although the cells had a definitive neuronal phenotype, the use of a general cell death endpoint in these cultures did not allow specific identification of neurotoxicants. As alternative approach, neurite growth was measured as an organ-specific functional endpoint. We found that neurite extension of developing LUHMES was specifically inhibited by diverse compounds such as colchicine, vincristine, narciclasine, rotenone, cycloheximide, or diquat. These compounds reduced neurite growth at concentrations that did not compromise cell viability, and neurite growth was affected more potently than the integrity of
\end{abstract}

\author{
A. K. Krug $(\bowtie) \cdot$ N. V. Balmer · F. Matt · M. Leist \\ Doerenkamp-Zbinden Chair for In Vitro Toxicology and \\ Biomedicine, University of Konstanz, Universitätsstr. 10, \\ Box 657, 78457 Constance, Germany \\ e-mail: anne.krug@uni-konstanz.de \\ F. Schönenberger · D. Merhof \\ Interdisciplinary Center for Interactive Data Analysis, \\ Modellingand Visual Exploration (INCIDE), University \\ of Konstanz, Constance, Germany \\ F. Schönenberger \\ Bioimaging Center (BIC), University of Konstanz, \\ Constance, Germany
}

developed neurites of mature neurons. A ratio of the EC50 values of neurite growth inhibition and cell death of $>4$ provided a robust classifier for compounds associated with a developmental neurotoxic hazard. Screening of unspecific toxicants in the test system always yielded ratios $<4$. The assay identified also compounds that accelerated neurite growth, such as the rho kinase pathway modifiers blebbistatin or thiazovivin. The negative effects of colchicine or rotenone were completely inhibited by a rho kinase inhibitor. In summary, we suggest that assays using functional endpoints (neurite growth) can specifically identify and characterize (developmental) neurotoxicants.

Keywords Neurite outgrowth - Compound screening · Developmental neurotoxicity

\section{Introduction}

Toxicological test systems do not only require initial conceptualization and basic description, as in other fields of science. They also necessitate detailed further development and a lengthy evaluation process. In some domains, such as cosmetics or drug testing, or in the pre-selection of environmental toxicants for more extensive testing, assays may be used without formal validation, if there is sufficient evidence for their scientific validity. Some regulatory authorities, as well as open platforms such as the evidencebased toxicology (EBT) consortium, provide guidance on method evaluation (Griesinger et al. 2009a; Hartung 2010). For instance, documents have been produced on good cell culture practice (GCCP) (Hartung et al. 2002), on guidelines for data presentation (Leist et al. 2010) and assay reliability (Schneider et al. 2009), on how to establish a test system for developmental neurotoxicity (DNT), and on 
how to select compounds for DNT testing (Kadereit et al. 2012; Kuegler et al. 2010; Zimmer et al. 2011). However, until now, only few test systems in the field of neurotoxicity and DNT have been developed further on the basis of such guidance documents (Bal-Price et al. 2012; Fritsche et al. 2011). More of this type of work is necessary, as it has recently been noted that several published studies are necessary for an evaluation of a method according to criteria of evidence-based toxicology (Judson et al. 2013; Stephens et al. 2013).

DNT often manifests itself in functional disturbances that may appear hard to model in vitro (van Thriel et al. 2011). However, it is widely assumed (Bal-Price et al. 2012; Hogberg et al. 2009; Kadereit et al. 2012) that DNT is ultimately the consequence of the disturbance of relatively basic biological processes, such as differentiation, proliferation, migration, and neurite growth. Therefore, several in vitro systems have been established that test the disturbance of such biological activities by chemicals (Balmer et al. 2012; Frimat et al. 2010; Harrill et al. 2011b; Radio et al. 2008; Zimmer et al. 2012). One endpoint that has found a lot of attention is neurite outgrowth (Radio and Mundy 2008). This activity is required during the formation of the nervous system for the development of dendrites and axons, and it is a precondition for synaptogenesis and cell connectivity. Different neuronal cell lines of human or rodent origin can be used to study neurite outgrowth and to measure disturbances after exposure to toxicants (Harrill et al. 2011a). A particular challenge for the development of neurite outgrowth assays is the evaluation of test system predictivity by comparison to in vivo data. As alternative, it has been suggested to focus more on data quality and on a broad evaluation of the biological basis of the test and its mechanistic consistency under many different situations and types of challenge (Leist et al. 2012).

From animal studies, it is known that chemicals can affect neurite growth in different ways. For instance, utero cocaine exposure reduced the total length of neurites in the locus coeruleus of rats (Snow et al. 2001). The pesticide diazinon impaired neurite outgrowth in the forebrain and brainstem of rats, exposed to the chemical on postnatal days 1-4 (Slotkin et al. 2006). Inhibited neurite formation was also observed after exposure of 7-day-old rat pups to ethanol (Joshi et al. 2006). In contrast, accelerated growth was observed, e.g., after treatment with the rho kinase (ROCK) inhibitor Y-27632 which enhanced the sprouting of corticospinal tract (CST) fibers after CST lesion in adult rats (Fournier et al. 2003).

Also in humans, disturbed neurite growth is one of the assumed reasons for disorders of neural development such as autism spectrum disorders (ASD). In adults with ASD, decreased axonal length has been observed postmortem in the anterior cingulate cortex (Zikopoulos and Barbas
2010). Moreover, numerous ASD candidate genes are linked to neurite outgrowth and neurite guidance (Hussman et al. 2011).

The number of in vivo studies analyzing the altered growth of neurites under toxicant stress is limited. It is still a technical challenge to visualize neurites in the developing or mature brain and to measure changes in the growth rate. New technologies for estimating neurite density in vivo (Vestergaard-Poulsen et al. 2011; Zhang et al. 2012) are currently under development but their application for investigating neurite toxicity in vivo still needs to be refined. A few studies make use of certain anatomical situations more suitable for analysis. From these, we know that PCBs can affect dendrite growth of dorsal root ganglion (DRG) neurons (Yang et al. 2009) and that different stress conditions affect dendrites in the hippocampus (McEwen 1999). Moreover, it is generally known that hypothyroidism during brain development affects neurite connections (Barakat-Walter et al. 2000). Apart from these pioneering neurodevelopmental studies, there is a large body of evidence that developed neurites are particularly sensitive targets of chemical toxicity. A large fraction of known neurotoxicants specifically targets neurites (Spencer et al. 2000). In such cases, specific neurite degeneration is often occurring independent of cell death. Prominent examples are chemotherapy-induced neuropathies (Quasthoff and Hartung 2002) after treatment with platinum compounds or alkaloids such as colchicine or vincristine. Another chemical class known to induce axonal neuropathy are acrylamide and related structures (LoPachin et al. 2002). The above findings suggest that neurites, developed or growing, play an important role in neurons. Therefore, there is a high need for human cellbased test systems that would provide data faster and easier than the hitherto used animal models.

Several test systems have already been developed successfully to assess neurite outgrowth in low density cultures (Harrill et al. 2010; Mitchell et al. 2007; Radio et al. 2008; Ramm et al. 2003; Yeyeodu et al. 2010), whereas the number of reports based on more interconnected high-density cultures is quite low (Narro et al. 2007; Stiegler et al. 2011; Wang et al. 2010), mainly due to the difficulties with assigning a specific neurite to a defined cell. A particular challenge for toxicological test systems for neurite growth is the definition of specificity of the observed effect for neurite growth. Identification of such compound features requires that generally cytotoxic effects are distinguished from the effects of chemicals that are specifically affecting neurite growth, but not overall cell survival. In the present study, we made use of a human cell-based high-density neuronal test system (Stiegler et al. 2011) to further explore the usefulness of simultaneous measurements of viability and neurite growth to define assay specificity. For this 
purpose, the system was challenged with a broad range of chemicals, including a high number of generally cytotoxic compounds. The dataset generated with the unspecific toxicants was found to be instrumental for the evaluation of assay performance with respect to the generation of false positives and for the identification of interesting true positive hits. The second goal of the study was to provide data on the performance characteristics and consistency of the assay under different types of challenge. For instance, we compared the toxicity of chemicals to developing versus developed neurites to answer the question whether a compound specifically inhibited the outgrowth of neurites. We also challenged the test system with groups of mechanistically, but not chemically, related compounds. The mechanistic consistency of the assay was further explored by exposing the test system to compound mixtures expected to behave additively or antagonistically.

\section{Materials and methods}

\section{Materials and chemicals}

Acrylamide, antimycin A, acetylsalicylic acid, blebbistatin, brefeldin A, buthionine sulfoximine (BSO), calceinAM, carbonyl cyanide 3-chlorophenylhydrazone (CCCP), chlorpyrifos, cisplatin, colchicine, cycloheximide, cytochalasin B, dibutyryl-cAMP (cAMP), 2,4-dinitrophenol, diquat dibromide, etoposide, fibronectin, fipronil, flavopiridol, hoechst bisbenzimide H-33342, honokiol, IPA3, potassium chromate $\left(\mathrm{K}_{2} \mathrm{CrO}_{4}\right)$, mannitol, menadione, methylmercury (II) chloride (MeHg), mevastatin, narciclasine, nocodazole, oligomycin, paraquat dichloride, puromycin, resazurin sodium salt, rotenone, saponin, sodium orthovanadate $\left(\mathrm{Na}_{3} \mathrm{VO}_{4}\right)$, SP600125, tert-butyl hydroperoxide $(\mathrm{tBuOOH})$, tetracycline, and vincristine were from Sigma (Steinheim, Germany).

Recombinant human FGF-2 and recombinant human GDNF were from R\&D Systems (Minneapolis). Bisindolylmaleimide I (Bis1), dimethyl sulfoxide (DMSO), 1H-[1,2,4] oxadiazolo[4,3- $\alpha$ ]quinoxalin-1-one (ODQ), okadaic acid potassium salt, PTP inhibitor IV, H1152, simvastatin, and U0126 were from Calbiochem (Darmstadt, Germany). Y-27632 was from Tocris Bioscience (Bristol, UK), tween-20 and sodium dodecyl sulfate (SDS) were from Roth (Karlsruhe, Germany), HA-1077 from Ascent scientific (Cambridge, UK), thiazovivin from Selleck (Munich, Germany), chlorpyrifos oxon from Chem Service Inc. (West Chester, USA), piericidin from Enzo life science (Lörrach, Germany), and methamphetamine from Lipomed (Arlesheim, Switzerland). All culture reagents were from Gibco unless otherwise specified.

\section{Cell culture}

Handling of LUHMES human neuronal precursor cells was performed as previously described in detail (Lotharius et al. 2005; Schildknecht et al. 2009; Scholz et al. 2011). Briefly maintenance of LUHMES cells was performed in proliferation medium, consisting of advanced DMEM/F12 containing $2 \mathrm{mM}$ L-glutamine, $1 \times \mathrm{N} 2$ supplement (Invitrogen), and $40 \mathrm{ng} / \mathrm{ml} \mathrm{FGF-2} \mathrm{in} \mathrm{a} 5 \%$ $\mathrm{CO}_{2} / 95 \%$ air atmosphere at $37{ }^{\circ} \mathrm{C}$. LUHMES cells were passaged every other day and kept until passage 20. For differentiation, 8 million cells were seeded in a Nunclon $\mathrm{T} 175$ in proliferation medium for $24 \mathrm{~h}$. The next day medium was changed to differentiation medium (DM II), consisting of advanced DMEM/F12 supplemented with $2 \mathrm{mM}$ L-glutamine, $1 \times \mathrm{N} 2,2.25 \mu \mathrm{M}$ tetracycline, $1 \mathrm{mM}$ dibutyryl $3^{\prime}, 5^{\prime}$-cyclic adenosine monophosphate (cAMP), and $2 \mathrm{ng} / \mathrm{ml}$ recombinant human glial cell-derived neurotrophic factor (GDNF). $48 \mathrm{~h}$ later, cells were trypsinized and seeded in a density of 100,000 cells $/ \mathrm{cm}^{2}$ on dishes precoated with $50 \mu \mathrm{g} / \mathrm{ml}$ poly-L-ornithine (PLO) and $1 \mu \mathrm{g} / \mathrm{ml}$ fibronectin in advanced DMEM/F12 containing $2 \mathrm{mM}$ L-glutamine, $1 \times \mathrm{N} 2$ and $2.25 \mu \mathrm{M}$ tetracycline but without cAMP and GDNF (DM).

\section{Standard experimental setup}

To detect effects on neurite growth, cells were seeded at a density of 30,000 cells per well in $50 \mu \mathrm{l} \mathrm{DM}$ on PLO/ fibronectin-coated 96-well dishes. Compounds were serially diluted in DM, and $50 \mu \mathrm{l}$ was added to the cells $1 \mathrm{~h}$ after seeding. Analyses were performed $24 \mathrm{~h}$ after initiation of the treatment. To detect effects on neurite degeneration, cells were seeded at the same density in $100 \mu \mathrm{l} \mathrm{DM}$. At day 5 (d5), DM was removed and $100 \mu \mathrm{l}$ of fresh DM with serially diluted compounds was added. Analyses were performed $24 \mathrm{~h}$ or $72 \mathrm{~h}$ later. The maximum DMSO concentration used was $0.33 \%$ and had no influence on cell viability or neurite growth.

\section{Resazurin measurement}

Cell metabolic activity was detected by a resazurin assay (Schildknecht et al. 2009). Briefly, $10 \mu \mathrm{l}$ resazurin solution were added to the cell culture medium to obtain a final concentration of $10 \mu \mathrm{g} / \mathrm{ml}$. After incubation for $30 \mathrm{~min}$ at $37^{\circ} \mathrm{C}$, the fluorescence signal was measured at an excitation wavelength of $530 \mathrm{~nm}$, using a $590 \mathrm{~nm}$ long-pass filter to record the emission. Fluorescence values were normalized by setting fluorescence values of untreated wells as $100 \%$ and the values from wells containing less than $5 \%$ calcein-positive cells as $0 \%$. 
Quantification of neurite outgrowth

Neurite growth was detected as previously described in detail (Stiegler et al. 2011). Briefly, cells were stained with $1 \mu \mathrm{M}$ calcein-AM and $1 \mu \mathrm{g} / \mathrm{ml} \mathrm{H}-33342$ for $30 \mathrm{~min}$ at $37{ }^{\circ} \mathrm{C}$. An Array-Scan VTI HCS Reader (Cellomics, PA) equipped with a Hamamatsu ORCA-ER camera was used for image acquisition. Ten fields per well were imaged in two channels using a $20 \times$ objective $(2 \times 2$ pixel binning). Excitation/emission wavelengths of $365 \pm 50 / 535 \pm 45$ were used to detect H-33342 in channel 1 and $474 \pm 40 / 535 \pm 45$ to detect the calcein signal in channel 2.

Nuclei were identified as objects in channel 1 according to their size, area, shape, and intensity. The nuclear outlines were expanded by $3.2 \mu \mathrm{m}$ in each direction, to define a virtual cell soma area (VCSA) which was bigger than the average cell size to reduce false positive neurite areas. All calcein-positive pixels of the field were defined as viable cellular structures (VCSs). In an automatic calculation, the VCSAs, defined in the H-33342 channel, were used as filter in the calcein channel and subtracted from the VCS. The remaining pixels (VCS-VCSA) in the calcein channel were defined as neurite area.

\section{Statistics and data mining}

Data are presented, and statistical differences were tested by ANOVA with post hoc tests as appropriate, using GraphPad Prism 5.0 (Graphpad Software, La Jolla, USA).

\section{Results and discussion}

Conditions and acceptance criteria for the use of neurite growth as test endpoint

LUHMES cells can be differentiated by the addition of tetracycline within 5 days to mature neurons, as evaluated by the expression of neuronal markers, by changes of their morphology, and by measurements of electrical activity (Scholz et al. 2011). It has been shown earlier that the cells start expanding their neurites on day 2 (d2) and that quantification of the overall neurite area at day $3(\mathrm{~d} 3)$ is a suitable measure of initial neurite growth (Stiegler et al. 2011). After 4-5 days, this growth is saturated, and a 'mature' neurite network of relatively constant size is established (Scholz et al. 2011). We used these characteristics here for two different test protocols: exposure to chemicals from $\mathrm{d} 2$ to $\mathrm{d} 3$, and measurement on $\mathrm{d} 3$ as parameter to assess 'neurite growth', and exposure to chemicals from day 5 (d5) to day 6 (d6), and measurement on d6 as parameter to assess 'neurite toxicity' (Fig. 1a). In a first, rough approximation, these two measures were assumed to reflect DNT (prevention of neurite formation) vs. neurotoxicity, i.e., damaging effects of compounds to already developed neurites. We are aware of the fact that such a strict classification represents a strong simplification of reality. Nevertheless, we assume that comparison of the two assays helps to identify compounds that act by inhibiting the growth (development) of neurites without having adverse effects on established neurite structures as such.

The hazardous effect of chemotherapeutic alkaloids, such as colchicine, vincristine, or of nocodazole on neurites is well established. These microtubule disruptors do not only interfere with the microtubule organization during cell division, but also with the extension of microtubules during axonal growth (Daniels 1972; Fontaine-Lenoir et al. 2006; Geldof et al. 1998). They were therefore considered here as a potential positive control to illustrate the assay algorithm. The measurement of neurite area is based on a life-cell staining of the total cell cytoplasm with calcein and imaging of the result on an automated microscope. The algorithm identifies then all live structures not belonging to a cell body as viable neurites, as illustrated in Fig. 1b. The example images of colchicine $(5 \mathrm{nM})$ effects demonstrate clearly that the compound reduced the neurite area, while the cell bodies were all still viable. An important feature of the assay is that it allows for a simultaneous quantification of viable cells (calcein-positive cells) out of the total cell number (all Hoechst 33342-stained nuclei) and the assessment of neurite growth (Fig. 1b).

Three microtubule inhibitors, that are structurally diverse and that show different affinities for tubulin, were used to test the sensitivity and reproducibility of the test system. Colchicine, nocodazole, and vincristine were tested over a large concentration range in three different cell preparations. The data showed reproducibly an inhibition of neurite growth and a potency ranking that corresponded to the ranking of tubulin affinities (Correia and Lobert 2001) (Fig. 1c). A key question is whether these neurite data can be used as such for the identification of developmental toxicants and/or their ranking in our assay system. Can compounds such as colchicine and vincristine really be considered developmental toxicants on the basis of such neurite growth data? We felt that such an interpretation would produce too many false positives and that additional criteria would be required to increase the specificity of the assay. This was examined from different angles in the following experiments.

The main confounding factor of neurite growth tests may be the effects of compounds on overall cell survival (named here: general viability). For instance, simple detergents (not assumed to be developmental toxicants or neurite toxicants) may produce similar neurite area curves as the apparently specific microtubule inhibitors. For this 
A

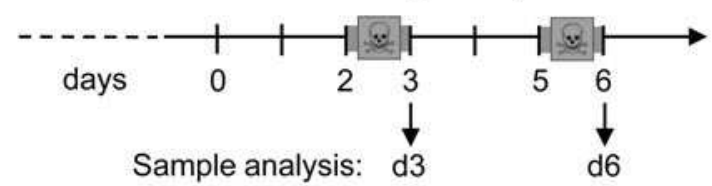

B
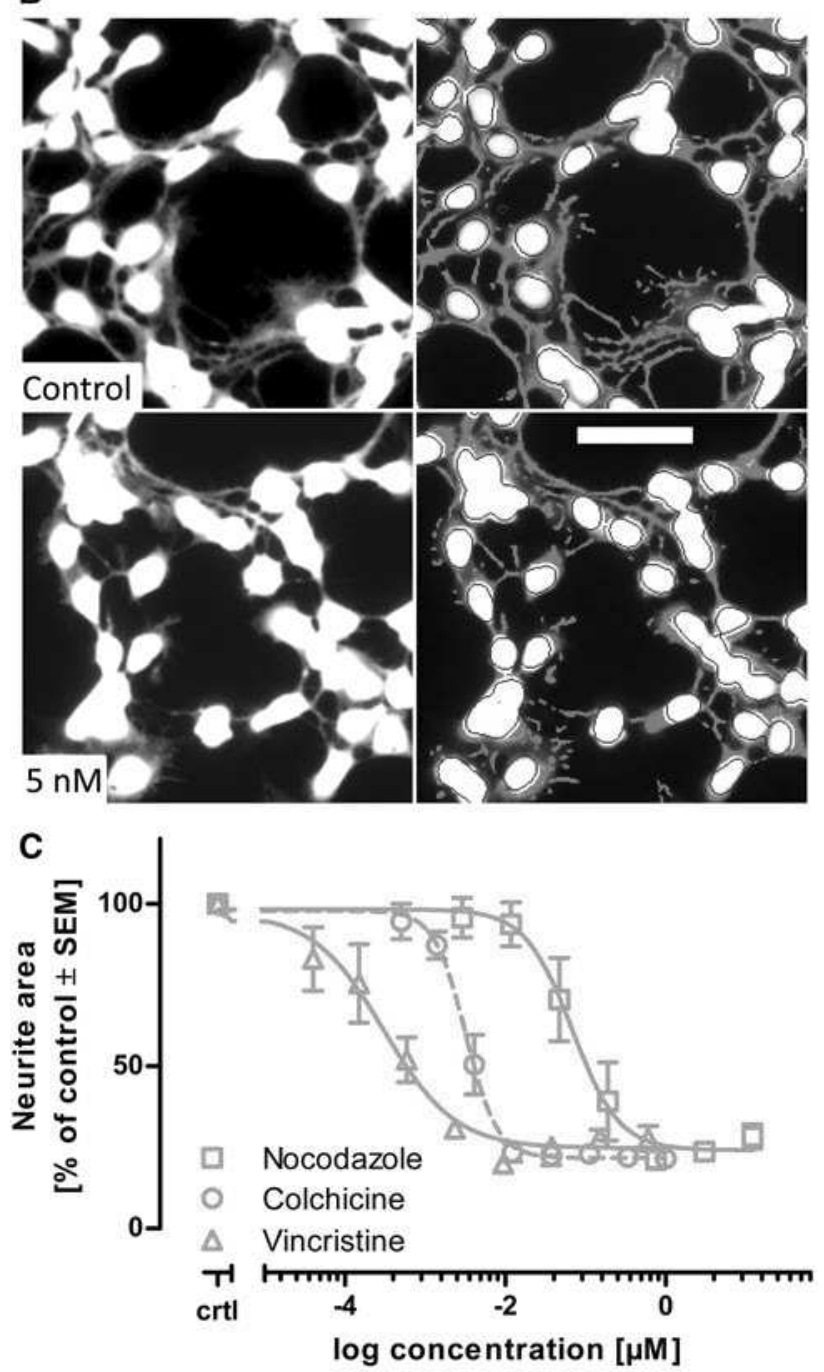

D

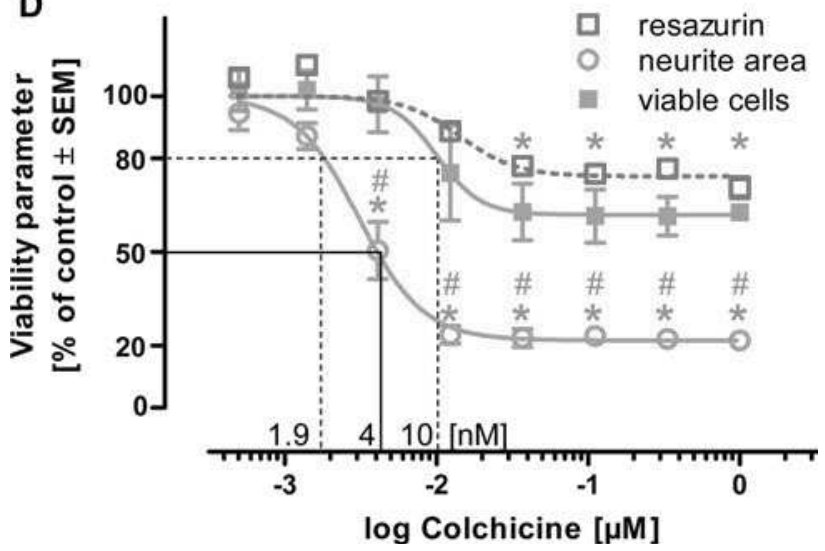

4 Fig. 1 Effect of microtubule-depolymerizing agents on neurite growth. Cells were replated at day 2 (d2) into 96-well dishes, and toxicants were added $1 \mathrm{~h}$ or 3 days later. At $24 \mathrm{~h}$ after the start of the incubation with chemicals, cells were stained with calcein-AM and $\mathrm{H}-33342$. The number of viable cells/field and the total neurite area/ field were automatically detected and quantified on a high-content screening microscope. a Exposure scheme of LUHMES cells. Cells were either treated on $\mathrm{d} 2$ for $24 \mathrm{~h}$ and endpoints were assessed on d3 (developing cells) or cells were differentiated until d5, treated for $24 \mathrm{~h}$ and measured on $\mathrm{d} 6$ (mature cells). b The upper row shows representative calcein images on the left side and the corresponding neurite area detected automatically by the imaging algorithm on the right side. The areas identified as neurites are marked in red; the nuclei of the cells detected by H-33342 staining are indicated by the circles. The lower row shows corresponding images of cells treated with $5 \mathrm{nM}$ colchicine. Scale bar $=50 \mu \mathrm{m}$. c Quantification of the neurite area of cells treated on $\mathrm{d} 2$ with nocodazole, vincristine or colchicine. d Colchicine was added to LUHMES on d2. Resazurin reduction was measured $23 \mathrm{~h}$ later. Subsequently, calcein-AM and H-33342 staining was performed to quantify the number of viable cells and the neurite area. Blue dashed lines indicate the EC20 values for neurite area $(1.9 \mathrm{nM})$ and viability $(10 \mathrm{nM})$, the black solid line indicates the EC50 of neurite area $(4 \mathrm{nM})$. All data points are mean \pm SEM from three independent experiments. ${ }^{*} p<0.05$ versus untreated control, ${ }^{\#} p<0.05$ versus viable cells at that concentration (color figure online)

reason, we assessed two general viability endpoints in all experiments in the same wells used for neurite evaluation: the relative number (=percentage) of viable cells and the capacity to reduce resazurin to resorufin. Colchicine was chosen again for an exemplary display. The comparison of all endpoints at many concentrations of the test compound showed that neurite growth is affected at much lower concentrations than the general viability. The EC20 for the neurite area was $1.9 \mathrm{nM}$, and for viable cells, it was $10 \mathrm{nM}$ (resulting in a ratio $>5$ ). The EC50 of the neurite area was $4 \mathrm{nM}$ and the viability was still $100 \%$ at that concentration (Fig. 1d). A reduction of neurite growth by $50 \%$, without reduction of viability, was also found for nocodazole and vincristine (suppl. Fig. 1a, b). Thus, on the basis of this complete set of data, all the three microtubule inhibitors can be considered as developmental neurotoxicants affecting neurite outgrowth.

To further explore the relationship of neurite growth and cytotoxicity, we chose a small set of diverse compounds for further testing. Etoposide, a topoisomerase inhibitor anti-cancer drug, and buthionine sulfoximine (BSO), a metabolic inhibitor of glutathione synthesis, were chosen as chemicals supposed not to interfere with neurites. Both compounds reduced neurite growth to a significant extent compared to untreated control cells, and the curve shape of neurite area did not look much different from that found for the microtubule inhibitors. When the 'apparent neurite growth inhibition' was compared with the reduction of viability, it became evident that the concentration 
Fig. 2 Comparison of compounds affecting neurite growth specifically or unspecifically. LUHMES cells were treated as in Fig. 1a; all compounds were added on $\mathrm{d} 2$ and effects were measured $24 \mathrm{~h}$ later. All data points are mean \pm SEM from 3 independent experiments. a Etoposide. b Buthionine sulfoximine (BSO). c Cycloheximide. d Paraquat. ${ }^{*} p<0.05$ versus untreated control, ${ }^{*} p<0.05$ versus viable cells at that concentration

dependencies for both endpoints were the same (Fig. 2a, b). This was not an averaging effect due to the combination of results from different test runs, but it was observed in each of at least three independent experiments (suppl. Fig 1c, d). The same data as for 'viable cells' were also found with the resazurin assay. In fact, the two tests were used for all experiments in this work, but as the results did not significantly differ, only one of the endpoints is indicated in most figures. We interpret the findings with BSO and etoposide in a way that 'apparently inhibited neurite growth' is a secondary consequence of reduced viability, and we suggest classifying such compounds as 'unspecific toxicants'.

This concept has a technical and a conceptual implication. Technically speaking, unspecific toxicants are neither classical negative (having no effect on the endpoints of the test system) nor positive hits (showing a specific effect). They form a group of their own. Conceptually, such compounds have to be interpreted as negative, i.e., as not affecting neurite growth in any specific way. As this may lead to misunderstandings, it requires some further specification: such a negative statement does not imply that a compound is not a developmental toxicant. It only implies that positive evidence for such an activity cannot be found in this assay system. There is no way to determine whether (a) the compound directly inhibits neurite growth, and in parallel also reduces viability in this particular cell culture system, or whether (b) it primarily reduces viability and that reduced neurite growth is found because of ongoing cell death. In simple terms, the neurite data cannot be interpreted in a meaningful way, when they are associated with ongoing cell death. As in all test systems, the increase in specificity (by including comparison to viability) is accompanied by a decrease in sensitivity (inability to classify compounds as developmentally neurotoxic, when they affect cell viability). An example illustrates how changes in the test system may alter sensitivity: theoretically, cells of another system may be more robust and tolerate concentrations of, e.g., $2 \mu \mathrm{M}$ etoposide without loss of viability. If neurite growth inhibition in those other cells would be seen at the same concentration as in LUHMES cells $(50 \%$ at $2 \mu \mathrm{M})$, this other test system would allow the detection of a DNT potential of etoposide that is masked in the LUHMES model by parallel cytotoxicity.
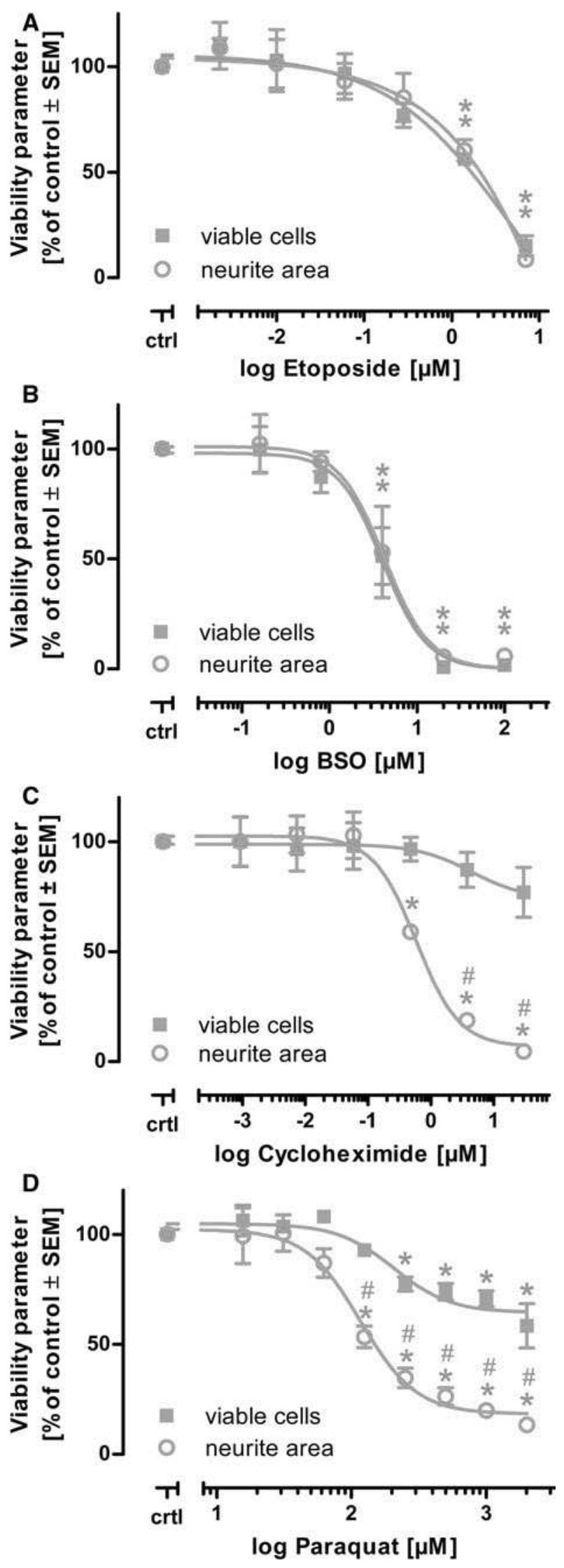
Testing of two further compounds with supposed effects on neurites indicated the need for some quantitative definition of specificity criteria to define a positive test result. Cycloheximide, an inhibitor of protein biosynthesis with strong effects on peripheral neurites (Gilley and Coleman 2010), reduced neurite growth significantly at concentrations at which no effect on viability was observed (Fig. 2c). There was a large ratio of the two endpoints of the EC20 values and EC50 values. However, the EC50 for general viability was not reached at testable compound concentrations. For ratio formation, we therefore introduced the rule that in this case, the highest concentration tested would be used for further calculations. Paraquat is a pesticide with potential toxicity for dopaminergic neurons (McCormack et al. 2002), and it affected neurite growth of LUHMES more potently than general viability (Fig. 2d). However, we observed some cytotoxicity at all concentrations associated with strongly reduced neurite growth. This is admittedly a case that may be classified as positive (specific developmental neurotoxicant) or negative (unspecific toxicant) depending on the rules of the assay interpretation model. We decided here to focus mainly on the horizontal shift of the curves as anchor point for interpretations of the LUHMES assay. This criterion may need to be adapted as more information on the underlying mechanisms becomes available. The general usefulness for screen purposes was explored in the following with a large number $(>30)$ of compounds suspected to affect neurites.

Classification of substances as specific neurite growth inhibitors

To define thresholds of assay specificity, we used unspecific toxicants. Nine compounds were chosen according to the following rules: (a) they are not known to affect neurite growth, (b) their known mode of action and their chemical properties make it unlikely that they specifically affect the biology of neurite growth. The selected chemicals were the glutathione synthesis inhibitor buthionine sulfoximine (BSO), the mitochondrial uncouplers CCCP and 2,4DNP, the detergents SDS and tween-20, the heavy metal ion $\mathrm{K}_{2} \mathrm{CrO}_{4}$, the DNA-interacting compounds etoposide and $\mathrm{H}-33352$ and the oxidant tertiary butyl hydroperoxide $(\mathrm{tBuOOH})$. This set of chemicals was used as 'unspecific controls', i.e., to define non-specific outcomes of the neurite growth inhibition assay. For this purpose, we determined their EC50 values for neurite growth inhibition and for reduction of general viability, and the ratio of these EC50 values was calculated for each experiment and each compound. The average \pm standard deviation (SD) of all these ratios was $1.4 \pm 0.83$, i.e., neurites were on average affected by unspecific compounds at slightly lower concentrations than general viability. For defining criteria for 'positive responses', we used a rule commonly used for many analytical methods as guidance; we assumed that significant effects (of specific compounds) should be 3 SD away from the baseline (average of unspecific compounds). Thus, we defined a ratio of 4 as threshold/acceptance criterion for compounds we regarded as positive hits of the screen (Fig. 3). Typical positive controls known from previous studies (U0126, flavopiridol, brefeldin A, bisindolylmaleimide I, and sodium orthovanadate $\left(\mathrm{Na}_{3} \mathrm{VO}_{4}\right)$ (Harrill et al. 2010; Radio et al. 2008; Radio and Mundy 2008; Stiegler et al. 2011) had EC50 ratios far above 4. Compounds with an EC50 ratio $<4$ were defined as negative. This rule is the pivotal basis for conferring specificity to the assay, even though it may reduce its sensitivity. A negative classification in our assay means that there is no positive evidence for a neurite growth inhibition. It is not evidence of absence of such a property.

Using these criteria, we screened substances that we found likely to affect neurite growth because of their assumed primary mode of action or because of reports in the literature (Fig. 3, suppl. Fig. 2). The tested compounds comprised many biological activity groups like cytostatic drugs (cisplatin), redox cyclers/pesticides (paraquat, diquat), mitochondrial toxins (rotenone, antimycin A, oligomycin, piericidin), cytoskeleton toxicants (colchicine, okadaic acid, nocodazole, vincristine), acetylcholineesterase inhibitors (chlorpyrifos, chlorpyrifos oxon), and other substances like the neurotoxin acrylamide, the guanylyl cyclase inhibitor ODQ, the antipsychotic and possible teratogenic drug haloperidol, the stress kinase inhibitor SP600125, the HMG-CoA reductase inhibitors simvastatin and mevastatin, an inhibitor of protein tyrosine phosphatases PTP IV, the RhoA activator narciclasine, a group of rho kinase (ROCK) inhibitors (H-1152, HA-1077, thiazovivin), and the myosin II inhibitor blebbistatin.

The tested microtubule inhibitors colchicine, nocodazole, and vincristine were classified as neurite growth-specific toxicants. In the group of tested pesticides consisting of rotenone, paraquat, diquat, chlorpyrifos, and chlorpyrifos oxon, a clear positive effect was determined for rotenone, paraquat, and diquat. Another group of compounds influencing neurite growth were Rho/ROCK pathway modifiers. Some of them accelerated the neurite growth instead of inhibiting it.

The ratio of the EC50 values of neurite growth versus viability proved to be a useful classifier for compounds associated with a developmental neurotoxic hazard. The results shown here are based on average EC50 values derived from three biological replicates (independent experiments).

For more extensive screens, a more simplified procedure is desirable. Therefore, we examined how the classification would have looked like for individual experiments. Also 


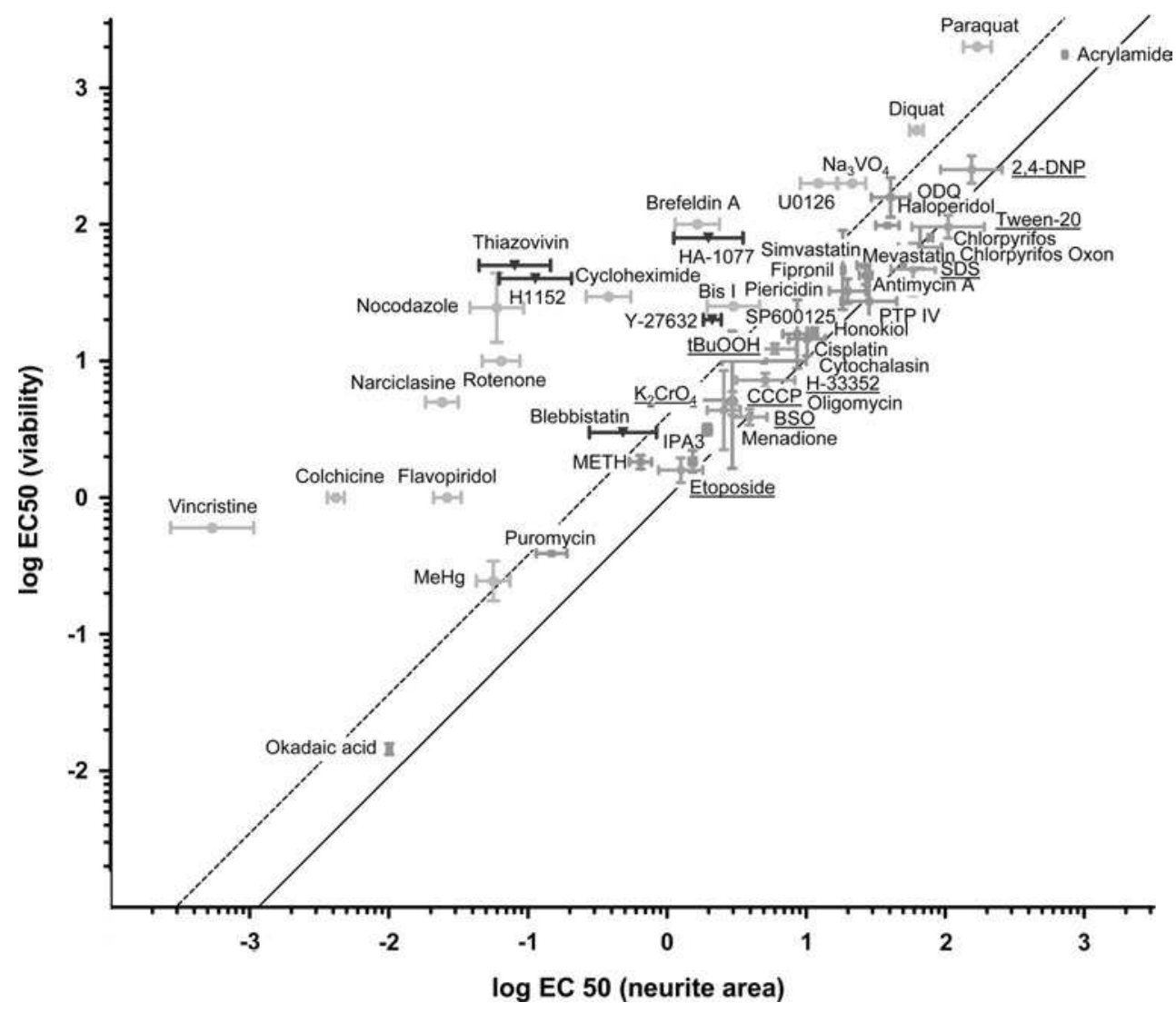

Fig. 3 Separation of specific neurite growth modulators from unspecific cytotoxicants. Cells were treated on $\mathrm{d} 2$ as displayed in Fig. 1a, and $24 \mathrm{~h}$ later, neurite area and viability were automatically quantified. Compounds were tested at several concentrations, and their EC50 values for effects on neurite area and cell viability were determined by a nonlinear regression sigmoidal concentration-response curve fit. The EC50 values of the neurite area were plotted against the EC50 values of general cell viability. First, a reference control group of 9 unspecific toxicants was measured (dots in gray, names are underlined). These comprised buthionine sulfoximine (BSO), carbonylcyanide-3-chlorophenylhydrazone (CCCP), 2,4-dinitrophenol (2,4-DNP), etoposide, bisbenzimide $\mathrm{H}$ (H-33352), potassium chromate (K2CrO4), tert-butyl hydroperoxide (tBuOOH), tween20 and sodium dodecyl sulfate (SDS). The solid line indicates equal EC50 of viability and neurite area. The dashed line indicates an EC50 ratio of 4. Data for 40 compounds were classified according to this

the data points of the positive compounds from individual experiments all fell within the 'specific area' of the scatter plot (suppl. Fig. 2). The approach taken here is firmly established in the field of biomolecular screening, as performed in pharmaceutical industry, but it differs from the traditional reporting of in vitro test systems in toxicology. The more traditional approach in this field is based on statistical evaluation of a compound effect vs. a negative control. The specificity definition we have chosen here is easily adaptable to other situations, including simpler assays with a single endpoint. Measures based in some way threshold value. Orange color indicates substances classified to act unspecific on neurite growth: acrylamide, antimycin A, chlorpyrifos, chlorpyrifos oxon, cisplatin, cytochalasin, fipronil, haloperidol, honokiol, IPA-3, menadione, methamphetamine (METH), mevastatin, $1 \mathrm{H}-[1,2,4]$ oxadiazolo-[4,3- $\alpha$ ]quinoxalin-1-one (ODQ), okadaic acid, oligomycin, piericidin, protein tyrosine phosphatase inhibitor IV (PTP IV), puromycin, simvastatin and SP600125. Substances classified as specific neurite growth inhibitors (light blue) were: bisindolylmaleimide I (Bis1), brefeldin A, colchicine, cycloheximide, diquat, flavopiridol, methylmercury (II) chloride (MeHg), sodium orthovanadate $\left(\mathrm{Na}_{3} \mathrm{VO}_{4}\right)$, narciclasine, nocodazole, paraquat, rotenone, U0126 and vincristine. Substances that increased the neurite area (dark blue) were as follows: blebbistatin, HA-1077, H1152, thiazovivin, and Y-27632. The 'neurite EC50' of these compounds was defined as the concentration resulting in a half-maximal increase in the neurite area. Data are mean \pm SD of 3 separate screens (color figure online)

on the variation/confidence limits of the reference group can always provide a useful tool to classify further tested compounds: either the compounds are within the 'noise limit' ( $\rightarrow$ negative classification) or outside the background noise ( $\rightarrow$ specific hits). In the LUHMES test system, we newly identified 7 specific neurite growth inhibitors (rotenone, narciclasine, colchicine, vincristine, nocodazole, paraquat, diquat) and 4 neurite growth accelerators (H1152, HA-1077, thiazovivin, blebbistatin). These results will be displayed and discussed in greater detail in the following sections. 
Fig. 4 Reduction of neurite growth by rotenone and other respiratory chain inhibitors. LUHMES cells were treated as in Fig. 1a. a Rotenone was added in fresh medium either $1 \mathrm{~h}$ after replating on $\mathrm{d} 2$ or on day 5 . After $24 \mathrm{~h}$ incubation, viability and neurites were measured and normalized to untreated controls. Viability curves of $\mathrm{d} 3$ and $\mathrm{d} 6$ were similar. Data are mean \pm SEM from 3 independent experiments. b Representative images are shown, in which the automatically detected neurite area (red) is overlaid over the calcein images. The position of the nuclei is marked by a blue outline. The width of the micrographs shown is $330 \mu \mathrm{m}$. Cells were incubated on $\mathrm{d} 2$ for $24 \mathrm{~h}$ with the indicated concentration of rotenone. c Cells were treated on $\mathrm{d} 2$ with rotenone for $24 \mathrm{~h}$. The data for viability and neurites are displayed for 4 independent experiments (dashed lines), each run in technical triplicates [individual error bars $( \pm \mathrm{SD})]$ ]. d Antimycin A or oligomycin was added to LUHMES after replating for $24 \mathrm{~h}$. Viability and neurite data are means from 2 (oligomycin) and 3 (antimycin A) independent experiments. All data points are mean \pm SEM from at least two independent experiments. ${ }^{*} p<0.05$ versus untreated control, ${ }^{\#} p<0.05$ neurite area versus viable cells at that concentration (color figure online)

Specific effects of rotenone, but not other respiratory chain toxicants

Rotenone, a complex I inhibitor of the mitochondrial respiratory chain, inhibited neurite growth significantly at $0.1 \mu \mathrm{M}$ (Fig. 4a, green solid line), whereas viability was affected only at ten times higher concentrations. This was initially surprising. To identify potential artifacts, the original images of the high-content screen were retrieved and evaluated by trained observers. The effect was fully confirmed, and representative example images show clearly that neurite area was reduced by low concentrations of rotenone, while the number of viable cells per field was not affected. Only at higher concentrations, a concentrationdependent decrease in cell number was observed, and all viable cells were completely devoid of neurites (Fig. 4b). This big difference of effects on neurite growth and viability was observed in four independent experiments (Fig. 4c). To follow up on this positive hit, we asked the question whether rotenone targets neurites in general or whether it specifically influences their growth. Therefore, we compared the effects of rotenone ( $24 \mathrm{~h}$ exposure in both cases) on differentiating LUHMES on day 2 (d2) with its effects on mature cells with a fully differentiated neurite network on day 5 (d5) (Fig. 4a, green dashed line). The mature neurites were less sensitive to rotenone. In fact, the concentration-dependency of the neurite degeneration was not significantly different from the one for general viability, when mature neurons were used as model system (Fig. 4a, orange dashed line). The general cytotoxicity of rotenone was the same for $\mathrm{d} 2$ and $\mathrm{d} 5$ cells (Fig. 4a viability for d5, Fig. 4c viability for $\mathrm{d} 2$ ), and only the sensitivity of the neurites was different. Thus, rotenone is an example for a compound

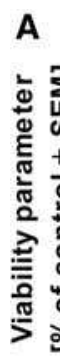

A
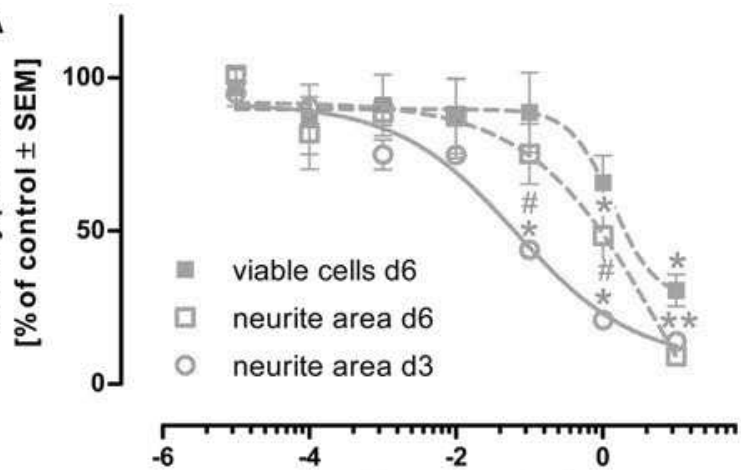

B
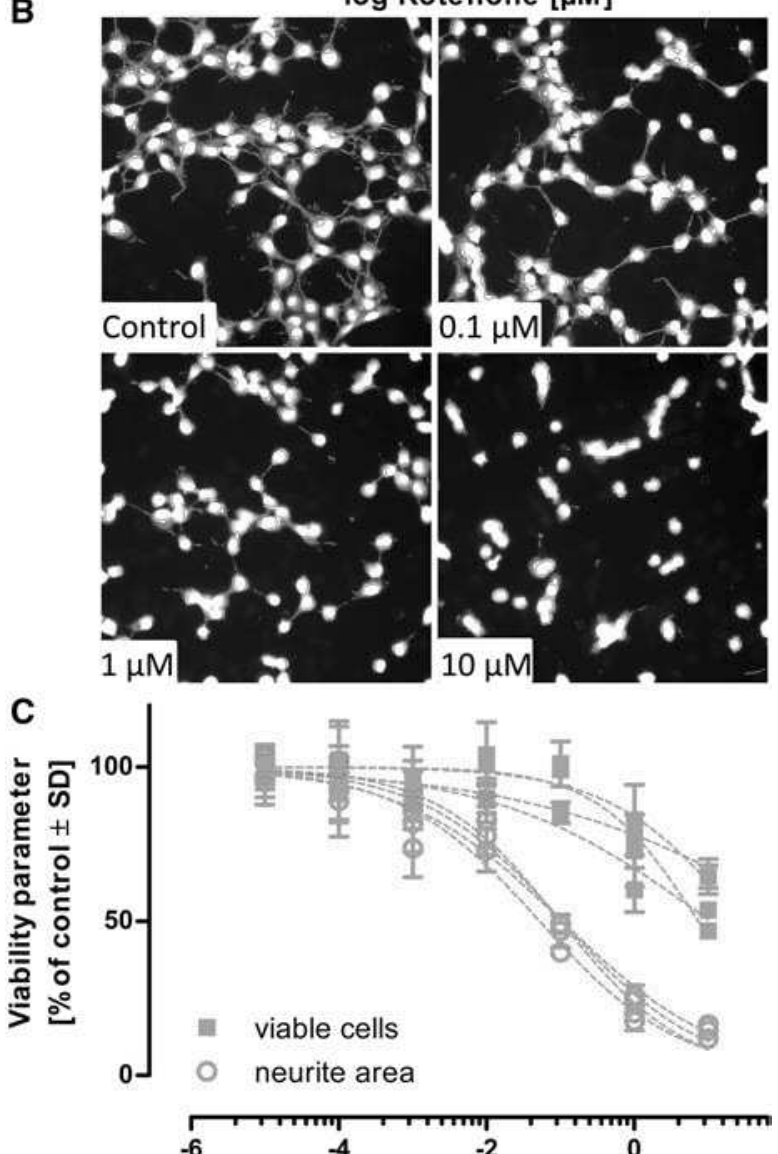

log Rotenone $[\mu \mathrm{M}]$

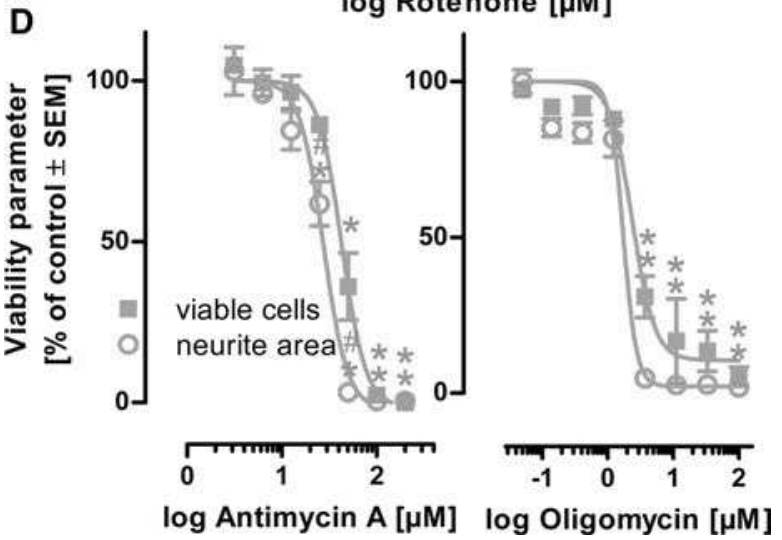


with a higher toxic potency for the developing neurons than for the developed cells. This effect was unique for rotenone, as we found no other mitochondrial toxicant with such an effect on neurites. Complex I inhibitor piericidin, complex V inhibitor oligomycin (Fig. 4d) as well as the uncouplers of oxidative phosphorylation CCCP or 2,4-DNP had no impact on neurites at several tested concentrations. For the complex III inhibitor antimycin A, we identified concentrations $(25-50 \mu \mathrm{M})$ at which neurites were significantly more affected than viability. But the EC50 ratio of viability to neurite area was only 1.6 (Fig. 4d), whereas rotenone showed a ratio $>15$. According to our rules, antimycin A was classified as negative.

Other reports, using rodent cells (PC 12 cells, primary hippocampal neurons), also suggest that rotenone has some specific effect on axon formation (Sai et al. 2008; Sanchez et al. 2007). The mechanism is unknown, but it has been suggested that complex I inhibitory parkinsonian toxicants may affect dopaminergic neurons by microtubule depolymerization (Ren et al. 2005). Other processes which are also dependent on correct microtubule formation like migration and proliferation have also been shown to be inhibited by rotenone in mesencephalic neural stem cells (Ishido and Suzuki 2010). The process of microtubule formation is indeed crucial for the growth of axons as suggested for instance by our findings on colchicine and related compounds. To identify the underlying mechanisms of rotenone's DNT more clearly, in-depth experiments and additional technical approaches are needed. As neurons can tolerate a partial depletion of ATP for long times, if secondary apoptotic processes are blocked (Poltl et al. 2012; Volbracht et al. 1999), a specific cell death-independent action of rotenone on young developing neurons seems likely.

Differential chemical effects on neurite growth versus neurite stability

Our observation that rotenone specifically targets neurite growth ( $\mathrm{d} 2$ cells), as compared to neurite stability (d5 cells), suggests that such a distinction may be used more generally to define the specificity of an assay (or a compound) for neurite growth inhibition. For this purpose, we tested a group of eleven compounds, which had been classified as specific neurite toxicants in the $\mathrm{d} 2 \rightarrow \mathrm{d} 3$ neurite growth assay, on neurite degeneration $(\mathrm{d} 5 \rightarrow \mathrm{d} 6)$ (Fig. 1a). Concentration-response curves were obtained for effects on the neurite area and general viability for d5 cells treated for $24 \mathrm{~h}$. Scatter plots of the effects on EC50 (general viability) versus EC50 (neurite area) measured on d3 (Fig. 5a) or d6 (Fig. 5b) showed that most compounds did not affect the mature neurites in a specific way (without inducing cell death). Nine of the eleven compounds were located on the

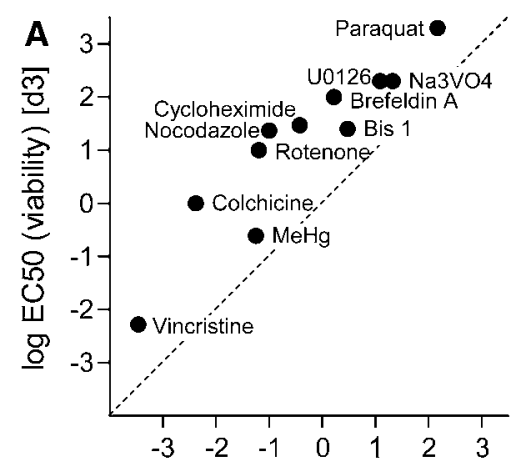

$\log$ EC50 (neurite area) [d3]

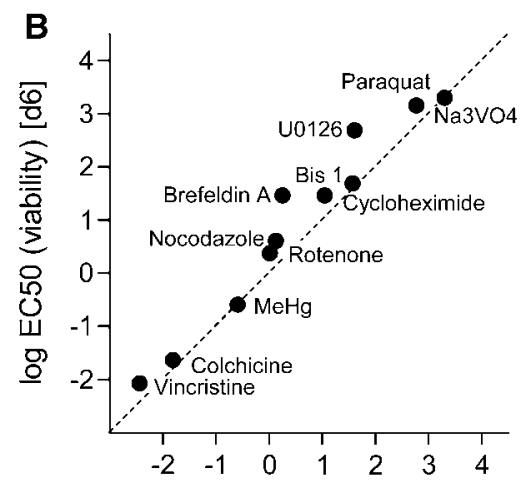

$\log$ EC50 (neurite area) [d6]

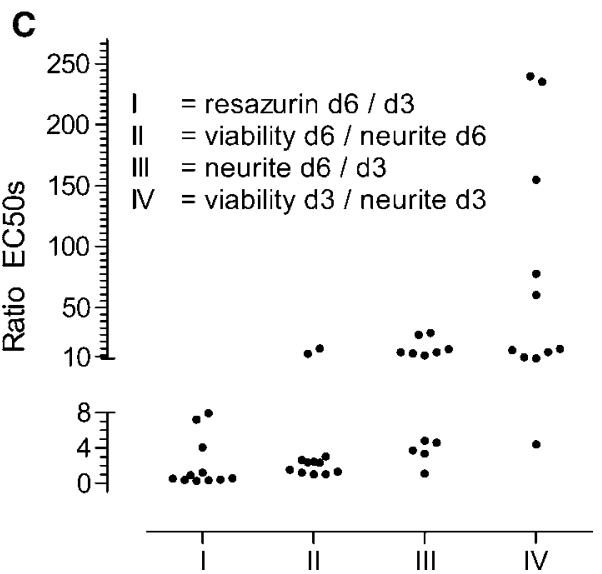

Fig. 5 Comparison of toxicant effects on d3 and d6. Cells were replated at $\mathrm{d} 2$ and eleven compounds were tested with at least five different concentrations on $\mathrm{d} 2$ or on $\mathrm{d} 5$. After $23 \mathrm{~h}$, resazurin reduction was measured. Subsequently, cells were stained with calcein-AM and $\mathrm{H}-33342$ for $30 \mathrm{~min}$. The number of viable cells and the neurite area were automatically detected by Cellomics Array Scan. EC50 values of neurite area were plotted against the EC50 s of viability. The dashed line indicates equivalent EC50 values of neurite area and viability. In cases of low cytotoxicity of compounds, the highest concentration measured was used as 'EC50 viability'. All data are means of 3 independent experiments. a Comparison of effects on viability and neurites on $\mathrm{d} 3$. b Comparison of effects on viability and neurites on d6. c Scatter plot of different EC50 ratios of the same compounds as in a/b. (1) EC50 ratio of resazurin reduction of d6 to d3, (2) EC50 ratio of calcein-positive cells to neurite area of d6, (3) EC50 ratio of neurite area of d6 to $\mathrm{d} 3$, (4) EC50 ratio of calcein-positive cells to neurite area of $\mathrm{d} 3$ 
dashed line, indicating identical EC50 values for both endpoints at $\mathrm{d} 6$.

The data obtained in these experiments also allowed to answer the question, whether the EC50 values for neurites or for general viability were shifted in absolute terms between $\mathrm{d} 3$ and $\mathrm{d} 6$ cells. Seven of the compounds were much more potent on developing neurites, than on developed neurites, and the average of the ratios of EC50 (neurites d6)/EC50 (neurites d3) was 11.4. This means that the functional endpoint of neurite growth is more sensitive toward toxicant exposure (Fig. 5c and suppl. Fig. 3a). To test whether developing cells are in general more sensitive to toxicant exposure than mature cells, we compared the EC50 values of resazurin reduction. The ratio of this endpoint for the two developmental stages of $d 6$ to $d 3$ is 0.74 . This suggests that the general cytotoxicity is independent of the developmental stage of the cells and that the younger cells are not less robust than adult cells (Fig. 5c and suppl. Fig. 3b).

The above data suggest indirectly that general cytotoxicity data are no good predictor for neurotoxicity, even though they are obtained from neuronal cultures. To examine this point in more detail, we selected a subgroup of our test compounds. They comprised neurotoxicants such as $\mathrm{MnCl}_{2}$, acrylamide, and trimethyltin chloride, as well as neurite growth inhibitors and non-neurotoxicants for which literature values could be found in the Halle registry of cytotoxicity data (Halle 2003). Resazurin reduction of d3 (suppl. Fig. 4a) and of d6 cells (suppl. Fig. 4b) was plotted against the data from the Halle registry, which are based on average cytotoxicity tests on several non-neuronal human cell lines such as HeLa and HEK293. The LUHMES cytotoxicity data and the Halle registry values correlated to about $85 \%$. This means that the cytotoxicity of compounds determined in young or mature LUHMES as test system correlates to a high degree with that observed in other human cell lines of non-neuronal origin. This corroborates our assumption that human neurotoxicity cannot be determined by cytotoxicity measurements in human neuronal cell cultures and that only a specific functional assay, such as neurite growth, yields specific results. Observations pointing into a similar direction were also made in other model systems (Gartlon et al. 2006).

Independence of key findings from data processing algorithm

Our concept of whole curve comparisons does not allow statements on individual concentrations of a given compound. Therefore, we were interested how individual test conditions (defined concentrations of defined compounds) would distribute in a scatter plot that correlates effects on neurites with those on general viability. We produced a

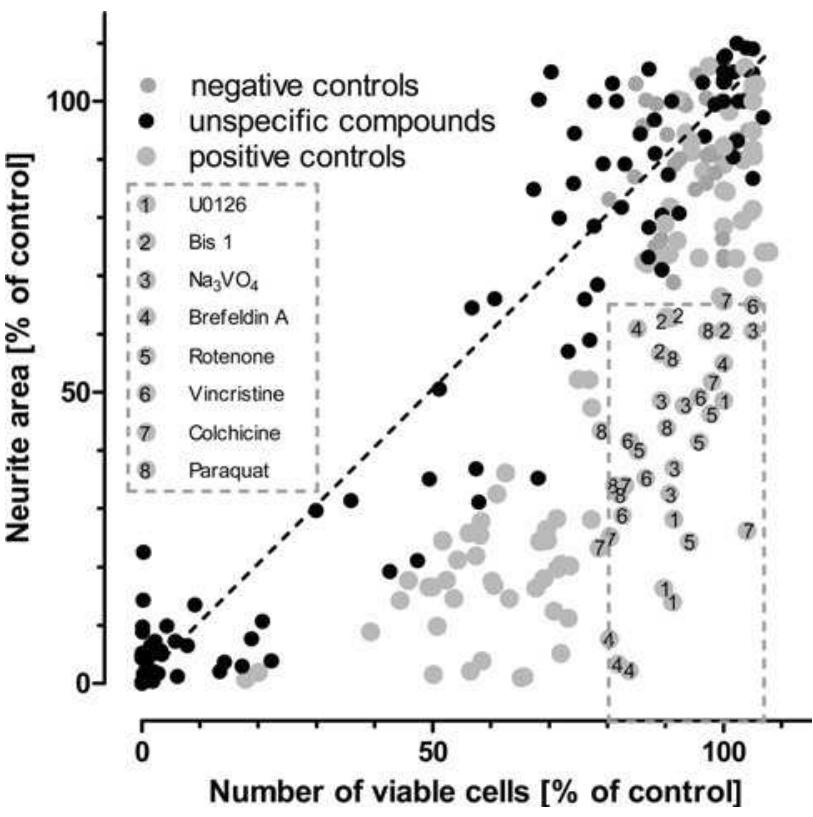

Fig. 6 Comparison of endpoint ratios (general viability vs. neurite area) of positive hits and unspecific toxicants at defined concentrations. LUHMES cells were treated and measured as in Fig. 3. Each concentration for each compound is represented by one individual dot in the scatter plot. The effects of substances on viability are plotted against effects on the neurite area. The dashed line indicates equivalent values for neurite area and viability. Negative controls, such as aspirin and mannitol, are marked by green dots. Black dots display values for unspecific compounds: buthionine sulfoximine (BSO), 2,4-dinitrophenol (2,4-DNP), etoposide, bisbenzimide H (H-33352), menadione, oligomycin, tert-butyl hydroperoxide (tBuOOH), tween20, saponin and sodium dodecyl sulfate (SDS). Data from specific compounds are marked by blue dots: bisindolylmaleimide I (Bis1), brefeldin $\mathrm{A}$, colchicine, cycloheximide, $\mathrm{MeHg}, \mathrm{Na}_{3} \mathrm{VO}_{4}$, nocodazole, paraquat, rotenone, U0126, and vincristine. The dashed gray box encircles dots which showed a reduction in neurite area of $>35 \%$ and in viability of $\leq 20 \%$

scatter plot of the individual data points for each concentration of a test compound, so that inhibition of neurite growth and the general cytotoxicity were used as coordinates. The data were plotted for three groups of compounds: negative controls, unspecific controls, and eight neurite growth inhibitors (Fig. 6). Negative controls were mannitol and acetylsalicylic acid. They did not affect any endpoint, even though concentrations up to $4 \mathrm{mM}$ for mannitol and $2 \mathrm{mM}$ for acetylsalicylic acid were chosen (Fig. 6, green dots). Unspecific compounds, like SDS, BSO, etoposide, oligomycin, $\mathrm{tBuOOH}$, affected neurite growth and viability to a more or less similar extent at all tested concentrations (Fig. 6, black dots). More detailed analysis shows that for such compounds concentrations exist, at which they reduce general cell viability significantly (by 30-60\%), but neurite area is reduced much more (by up to $35 \%$ more). Antimycin A, mentioned in the paragraph above (Fig. 4d), is also such a compound. It is important for the understanding 


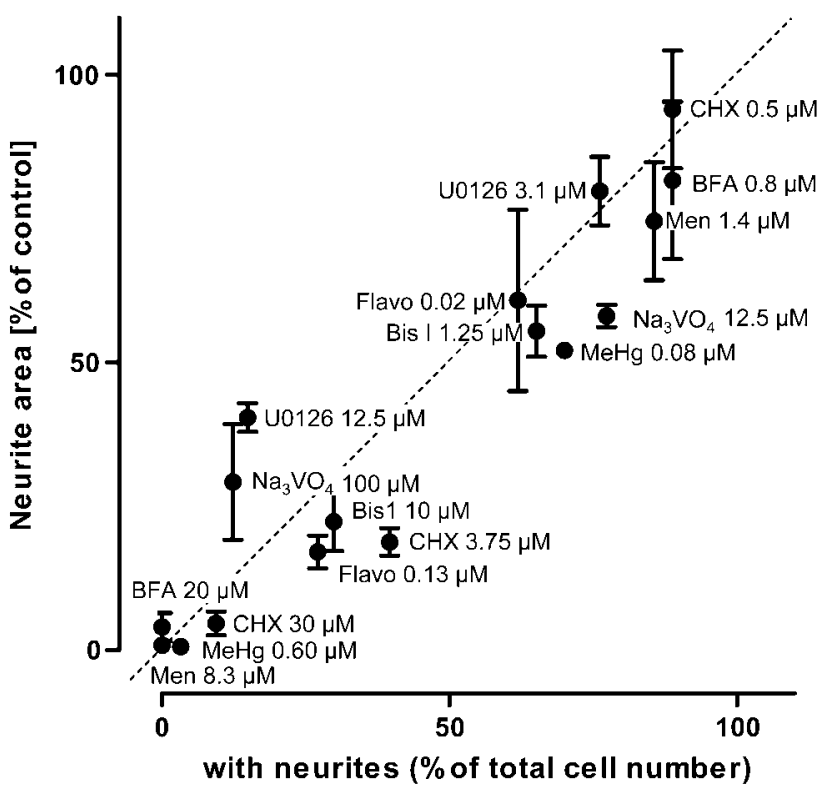

Fig. 7 Comparison of the field-based algorithm with a single cellbased readout. LUHMES cells were treated as in Fig. 1a. Incubations were started on $\mathrm{d} 2$ and ended $24 \mathrm{~h}$ later to assess neurite area and number of viable cells per field. Cells were treated with substances (BFA brefeldin A, Bis1 bisindolylmaleimide I, $C H X$ cycloheximide, Flavo flavopiridol, Men menadione) at the concentrations indicated, and neurite area was assessed automatically (by the field-based Cellomics algorithm). The same images were re-analyzed manually. Every individual cell was scored for having a neurite extension that was longer than the corresponding cell soma diameter or not. Data from the field-based algorithm ( $y$-axis) were compared with manually counted cells with neurites ( $x$-axis)

of our test approach that the specificity rule we used here classifies such substances as negative. The compounds classified as specific inhibitors of neurite growth localized differently in the scatter plot; there were always data points that showed a clear impact on neurites, with no major influence on viability (Fig. 6, blue dots). This way of data evaluation (based on different principles than the EC50 ratios) could form an alternative basis for a specificity rule. It appears to be useful as an option for smaller screens. Notably, the newly identified neurite growth inhibitors found in our screen would also have been detected based on these alternative criteria.

As a further control, we also examined whether other methods to quantify neurite effects would lead to similar results. For this purpose, we counted the percentage of cells with or without neurites for several representative experimental conditions and using the same images that had been used for the automated neurite area quantification algorithm. The neurite area endpoint correlated well with the number of cells with neurites obtained by manual counting (Fig. 7). A smaller number of conditions was also used for automated counting of neurite-containing cells, based on specifically developed software (Schoenenberger et al. 2012). Also in this case, the endpoints correlated well. We conclude from these comparisons that the toxicological effects we observed for neurite growth inhibitors in the LUHMES assay (as presented here) are robust and can be detected by different analytical methods as well.

\section{Detection of compounds that increase the neurite area}

An important parameter for each assay is its dynamic range. A particular question is whether deviations from normal can be measured into both directions and which types of positive controls can be used. The compound Y-27632 has been known to affect LUHMES neurite growth positively (Stiegler et al. 2011). These findings and other literature data (Fuentes et al. 2008; Kubo et al. 2008; Nikolic 2002) pointed to a role of the ROCK pathway in the control of neurite growth. The pathway is triggered by the activated RhoGTPase RhoA that binds to the rho kinase (ROCK) and activates it thereby. ROCK phosphorylates myosin light chain (MLC), and this results in the induction of actinactivated non-muscle myosin II ATPase. The downstream consequences are a local collapse of the neuritic growth cone and induction of stress fibers (Kubo et al. 2008). An inhibition of this pathway would therefore lead to an accelerated neurite growth due to less stress fiber formation and a reduced tendency of growth cone collapse. The role of this signaling cascade for our test system was explored further by the use of different compounds that affect this pathway. We found that the different ROCK inhibitors H1152, HA-1077, and thiazovivin as well as the myosin II inhibitor blebbistatin accelerated neurite growth significantly (Fig. 8a, c-e). The area of the culture dish covered with neurites was increased by up to $80 \%$ (with inhibitor HA-1077). When the same compounds were used on d5 LUHMES, no measurable effect was observed (data not shown). Inhibition of the ROCK pathway therefore seems to have a particularly prominent role in the growth process of neurites. Whether compounds leading to accelerated neurite growth should be interpreted as toxicants is an open issue and should be the subject of further investigations. In regenerative medicine, and in adults, accelerated outgrowth or preservation of neurites would rather be considered beneficial (Hansson et al. 2000; Schierle et al. 1999; Volbracht et al. 1999, 2001, 2006). There is, however, some published evidence that uncontrolled elongation of neurites during development may be related to neurotoxicity: hypertrophic dendritic outgrowth has been observed in parts of the embryonic prefrontal cortex after cocaine had been administered to pregnant rabbits at gestational stages (Jones et al. 2000; Stanwood et al. 2001).

We also investigated potentially toxic effects of the ROCK pathway activation. Narciclasine, which greatly 

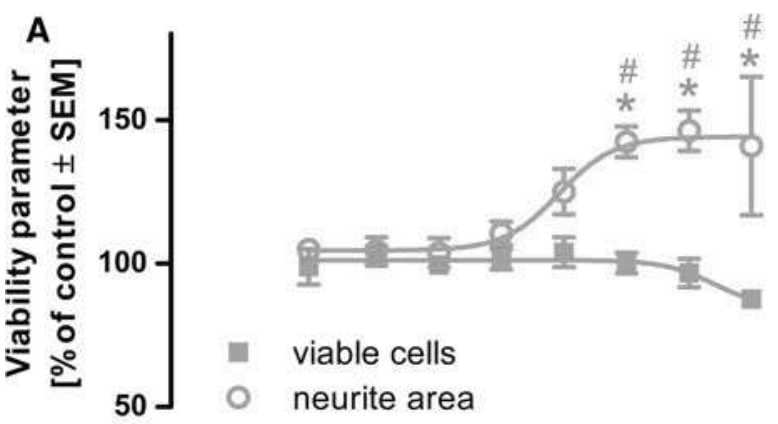

\section{viable cells \\ - neurite area}

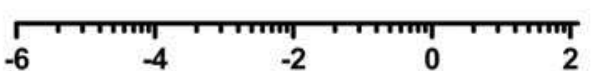

$\log$ Thiazovivin $[\mu \mathrm{M}]$
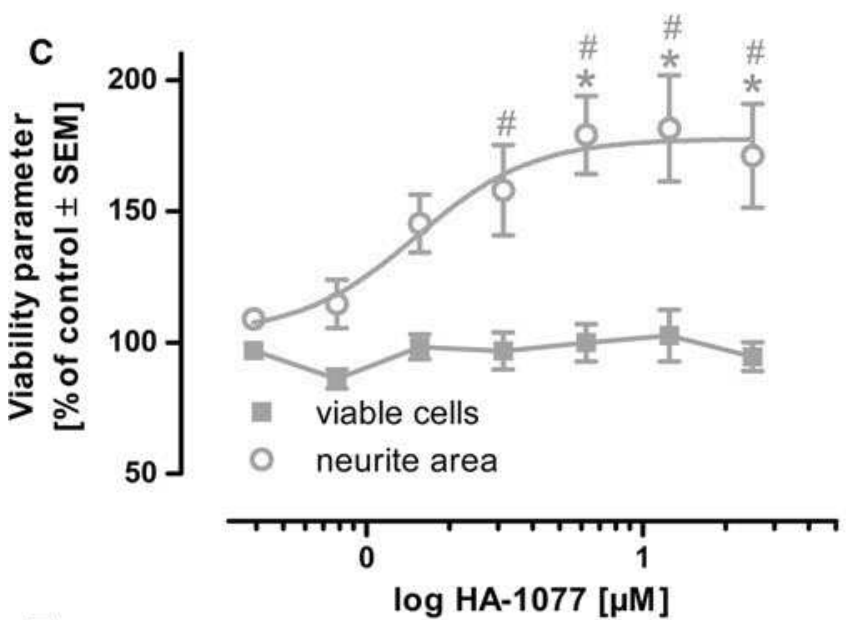

E

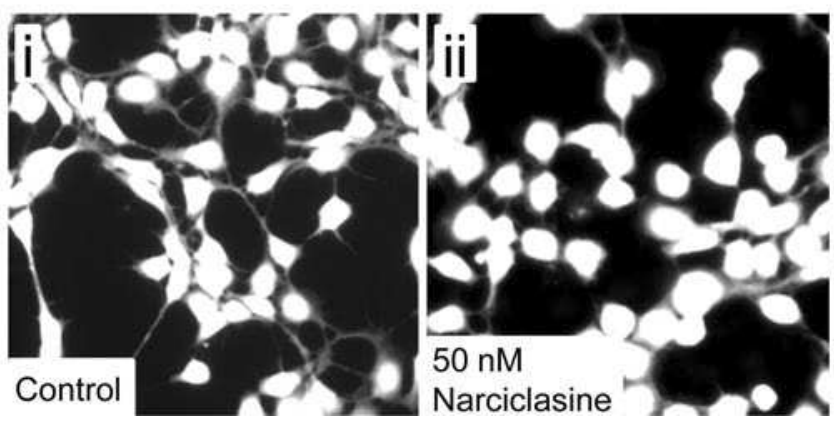

Fig. 8 Modulation of neurite outgrowth via the ROCK/RhoA pathway. At d2, cells were replated into 96-well plates and compounds were added at the concentrations indicated. At $24 \mathrm{~h}$ later, cells were stained with calcein-AM and H-33342 for $30 \mathrm{~min}$ at $37{ }^{\circ} \mathrm{C}$. Neurite area and viability were automatically detected using Cellomics

increases Rho A's activity (Lefranc et al. 2009), strongly decreased neurite growth (Fig. 8b, e). These data underline the mechanistic consistency of the assay, as the achieved results were as expected considering the interference of these compounds with the ROCK pathway. The fact that we can detect accelerating as well as inhibitory effects on neurite growth gives evidence of the broad dynamic range of our growth assay. Possibly, the test system may also be
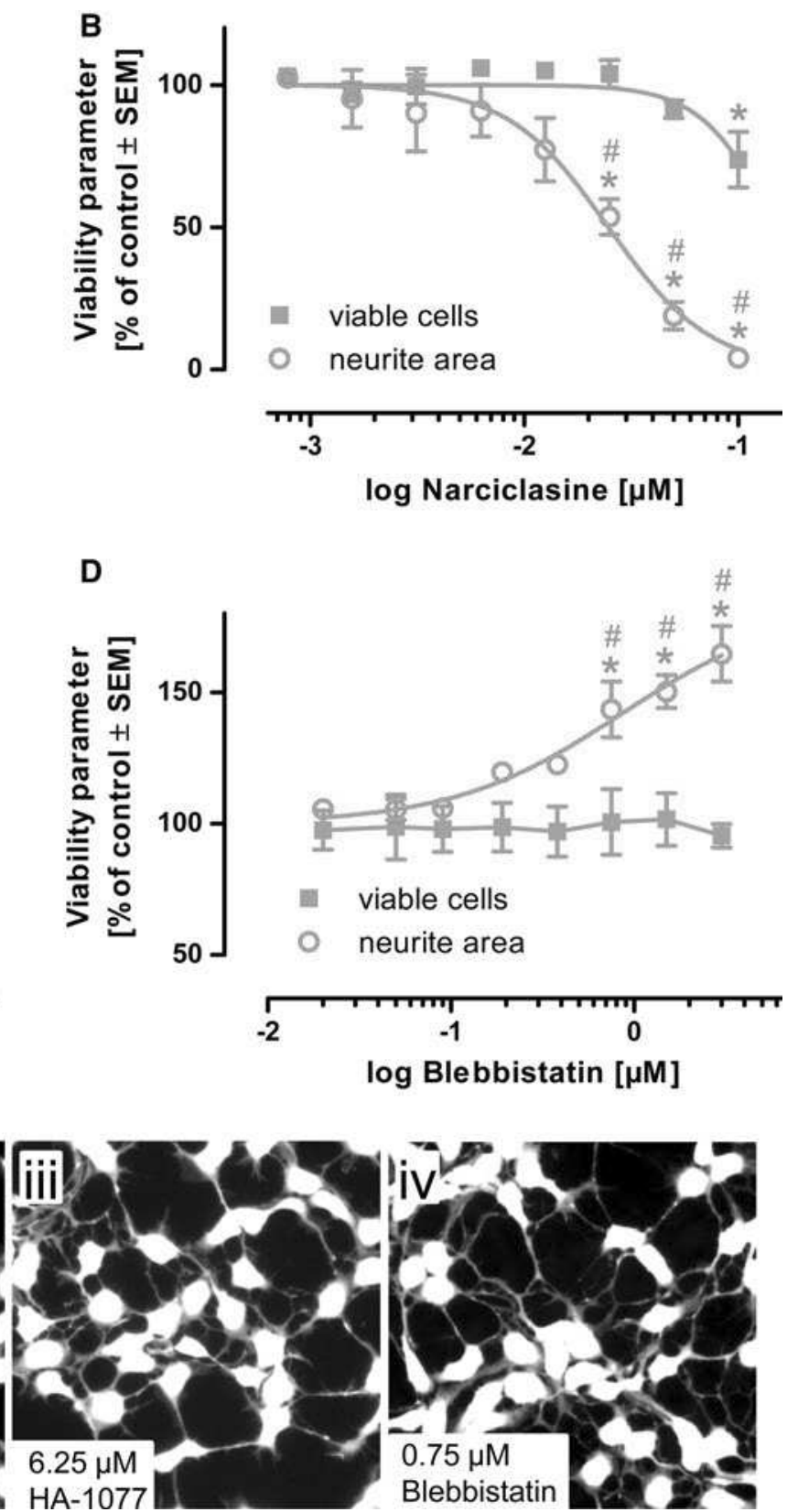

Array Scan. a Thiazovivin. b Narciclasine. c HA-1077. d Blebbistatin. e Representative micrographs (1) control, (2) narciclasine, (3) HA-1077, (4) blebbistatin. The width of each micrograph corresponds to $210 \mu \mathrm{m}$

used for pharmacological questions, e.g., for the identification of compounds that facilitate neuroregeneration by accelerating neurite growth.

Biological effects of combinations of substances

In the last step of the assay evaluation, we tested the effect of combinations of compounds. Consistent responses of the 
Fig. 9 Antagonistic and additive effects of different neurite growth modifiers. LUHMES cells were treated as in Fig. 1a. Incubations were started on $\mathrm{d} 2$ and $24 \mathrm{~h}$ later, the neurite area and the number of viable cells per field were assessed. a For all data points shown, $2 \mu \mathrm{M}$ of bisindolylmaleimide I (Bis1) was added. In addition, different concentrations $(0-12.5 \mu \mathrm{M})$ U0126 were added at the same time. The data for Bis1 alone are shown on the left part of the $x$-axis. b Combination of ROCK inhibitor Y-27632 plus $2 \mu \mathrm{M}$ Bis1. c Combination of ROCK inhibitor Y-27632 plus $5 \mathrm{nM}$ of colchicine. d Combination of ROCK inhibitor Y-27632 plus $0.1 \mu \mathrm{M}$ of rotenone. All data are mean \pm SEM of 3 independent experiments. Data are normalized to untreated controls (ctrl). $* p<0.05$ versus single compound treatment indicated on the left part of the $x$-axis

test system to at least binary mixtures would indicate its usefulness for more mechanistic questions and for exploring toxicity intervention. Moreover, we hoped to find additional evidence for the specificity of the hits discovered in our initial screen (Fig. 3). In a first set of experiments, we replicated earlier findings on the combination of the two kinase inhibitors bisindolylmaleimide I (Bis1) and U0126 (Stiegler et al. 2011). At certain drug concentrations, the neurite area could nearly be reduced to zero, without a significant reduction of cell viability. Moreover, three entirely independent experiments with the combination of two chemicals gave consistent results (Fig. 9a). We interpret this as indication for a high reproducibility and robustness of the test system.

A recent pilot study (Stiegler et al. 2011) indicated that the ROCK inhibitor Y-27632 is able to counteract the neurite growth inhibition of the MAP kinase (MAPK) inhibitor U0126 and that U0126 diminished the neurite accelerating effects of Y-27632. Such effects were now explored on a broader basis. We used the PKC inhibitor Bis1 to reduce neurite growth. Then, cells were co-exposed to eight different concentrations of the ROCK inhibitor Y-27632. A concentration of about $1 \mu \mathrm{M}$ of the ROCK inhibitor brought the neurite area back to $100 \%$ (from a low start level of $60 \%$ by using the PKC inhibitor alone), and concentrations of $10 \mu \mathrm{M}$ increased the neurite area to $130 \%$ of untreated controls (Fig. 9b). The potency of Y-27632 (half-maximal effect at about $2 \mu \mathrm{M}$ ) was similar to its potency, when used alone (Fig. 3). Interestingly, Y-27632 also counteracted the growth-decreasing effects of colchicine and of rotenone; the concentration of the ROCK inhibitor required to show significant effects was always in a similar narrow concentration range, and the set of experiments yielded highly reproducible data (Fig. 9c, d).

In these experiments, the toxic effects of rotenone and colchicine were neutralized by a treatment that supposedly promotes neurite growth, but does not affect the binding of the toxicants to the primary targets (tubulin or mitochondrial complex I). These findings suggest that the
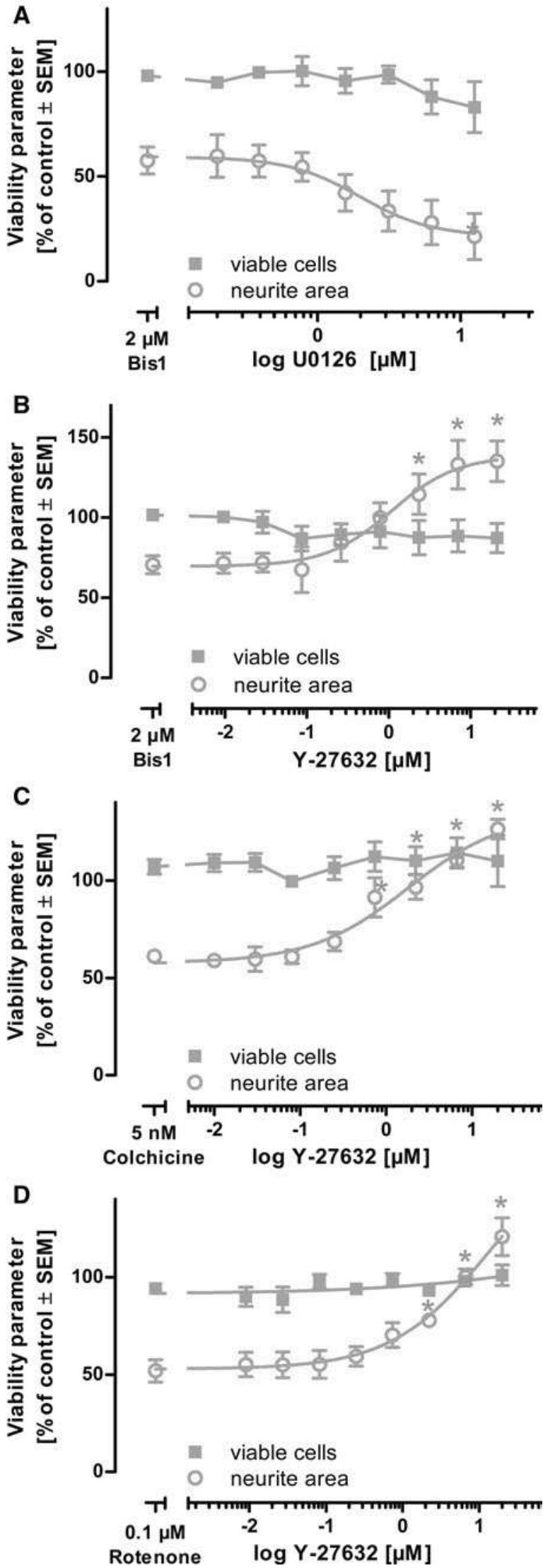
adverse outcome of toxic compound exposure may not only depend on the assumed molecular initiating event, but also on many other factors. However, this would require detailed investigation in a more mechanistically oriented study. There is in fact evidence from the literature that the ROCK pathway may affect microtubule stability (Gorovoy et al. 2005; Takesono et al. 2010). Some earlier data from other cellular systems suggest a rescuing effect of Y-27632 after treatment with rotenone (Sanchez et al. 2007) or with microtubule destabilizing compounds, such as colchicine or nocodazole (Keller et al. 2002; Niggli 2003; Zhang et al. 2001). These results from our test system corroborate such findings and indicate a good technical and mechanistic consistency of the test system. Intervention with toxicant effects would not only be helpful for clarifying the mode of action of DNT compounds, but it could also be interesting to explore potential rescue strategies after poisoning.

The practical application of toxicological in vitro test systems requires an extensive characterization of their performance characteristics. Especially, the regulatory use of new animal-free assays has been strictly coupled to a formal validation procedure, as performed, e.g., in Europe by the European Centre for Validation of Alternative Methods (ECVAM) (Corvi et al. 2012; Griesinger et al. 2009b). Before such a time- and resource-consuming validation is performed, it is now common practice to pre-validate, e.g., assay reproducibility and biological relevance. For highthroughput assays, evaluations similar to a formal pre-validation have been suggested as routine procedure to assess the usefulness and performance of the assays (Judson et al. 2013). In both cases, this step of assay establishment requires a broad range of data to be generated and multiple compounds to be used. This process usually goes far beyond an initial publication of a new test system (Hartung 2007, 2010; Leist et al. 2012). We have attempted here to provide such data and to provide a transparent and broad description of a test system that may be taken as example for similar approaches with other test systems.

Acknowledgments We are indebted to many colleagues for valuable contributions and insightful discussions during the course of this work. This work was supported by grants and support from the DoerenkampZbinden foundation, the German research foundation (research training group 1331), the European Commission's Seventh Framework Programme (ESNATS) and the German ministry for research (BMBF).

Conflict of interest The authors declare no conflict of interest.

\section{References}

Balmer NV, Weng MK, Zimmer B, et al (2012) Epigenetic changes and disturbed neural development in a human embryonic stem cell-based model relating to the fetal valproate syndrome. Hum Mol Genet 21(18):4104-4114
Bal-Price AK, Coecke S, Costa L et al (2012) Advancing the science of developmental neurotoxicity (DNT): testing for better safety evaluation. ALTEX 29(2):202-215

Barakat-Walter I, Kraftsik R, Kuntzer T, Bogousslavsky J, Magistretti P (2000) Differential effect of thyroid hormone deficiency on the growth of calretinin-expressing neurons in rat spinal cord and dorsal root ganglia. J Comp Neurol 426(4):519-533

Correia JJ, Lobert S (2001) Physiochemical aspects of tubulin-interacting antimitotic drugs. Curr Pharm Des 7(13):1213-1228

Corvi R, Aardema MJ, Gribaldo L et al (2012) ECVAM prevalidation study on in vitro cell transformation assays: general outline and conclusions of the study. Mutat Res 744(1):12-19

Daniels MP (1972) Colchicine inhibition of nerve fiber formation in vitro. J Cell Biol 53(1):164-176

Fontaine-Lenoir V, Chambraud B, Fellous A et al (2006) Microtubuleassociated protein 2 (MAP2) is a neurosteroid receptor. Proc Natl Acad Sci USA 103(12):4711-4716

Fournier AE, Takizawa BT, Strittmatter SM (2003) Rho kinase inhibition enhances axonal regeneration in the injured CNS. J Neurosci 23(4):1416-1423

Frimat JP, Sisnaiske J, Subbiah S et al (2010) The network formation assay: a spatially standardized neurite outgrowth analytical display for neurotoxicity screening. Lab Chip 10(6):701-709

Fritsche E, Gassmann K, Schreiber T (2011) Neurospheres as a model for developmental neurotoxicity testing. Methods Mol Biol 758:99-114

Fuentes EO, Leemhuis J, Stark GB, Lang EM (2008) Rho kinase inhibitors Y27632 and H1152 augment neurite extension in the presence of cultured Schwann cells. J Brachial Plex Peripher Nerve Inj 3:19

Gartlon J, Kinsner A, Bal-Price A, Coecke S, Clothier RH (2006) Evaluation of a proposed in vitro test strategy using neuronal and non-neuronal cell systems for detecting neurotoxicity. Toxicol In Vitro 20(8):1569-1581

Geldof AA, Minneboo A, Heimans JJ (1998) Vinca-alkaloid neurotoxicity measured using an in vitro model. J Neurooncol 37(2):109-113

Gilley J, Coleman MP (2010) Endogenous Nmnat2 is an essential survival factor for maintenance of healthy axons. PLoS Biol 8(1): 1000300

Gorovoy M, Niu J, Bernard O et al (2005) LIM kinase 1 coordinates microtubule stability and actin polymerization in human endothelial cells. J Biol Chem 280(28):26533-26542

Griesinger C, Hoffmann S, Kinsner A, Coecke S, Hartung T (2009a) Special issue: evidence-based toxicology (EBT). Preface. Hum Exp Toxicol 28(2-3):83-86

Griesinger C, Barroso J, Zuang V, Cole T, Genschow E, Liebsch M (2009b) Explanatory background document to the OECD draft test guideline on in vitro skin irritation testing. In: Organisation for economic co-operation and development (OECD). http://www. oecd.org/chemicalsafety/testing/43670220.pdf

Halle W (2003) The registry of cytotoxicity: toxicity testing in cell cultures to predict acute toxicity (LD50) and to reduce testing in animals. Altern Lab Anim 31(2):89-198

Hansson O, Castilho RF, Kaminski Schierle GS et al (2000) Additive effects of caspase inhibitor and lazaroid on the survival of transplanted rat and human embryonic dopamine neurons. Exp Neurol 164(1):102-111

Harrill JA, Freudenrich TM, Machacek DW, Stice SL, Mundy WR (2010) Quantitative assessment of neurite outgrowth in human embryonic stem cell-derived $\mathrm{hN} 2$ cells using automated highcontent image analysis. Neurotoxicology 31(3):277-290

Harrill JA, Freudenrich TM, Robinette BL, Mundy WR (2011a) Comparative sensitivity of human and rat neural cultures to chemical-induced inhibition of neurite outgrowth. Toxicol Appl Pharmacol 256(3):268-280 
Harrill JA, Robinette BL, Mundy WR (2011b) Use of high content image analysis to detect chemical-induced changes in synaptogenesis in vitro. Toxicol In Vitro 25(1):368-387

Hartung T (2007) Food for thought... on validation. ALTEX 24(2):67-80

Hartung T (2010) Evidence-based toxicology - the toolbox of validation for the 21st century? ALTEX 27(4):253-263

Hartung T, Balls M, Bardouille C et al (2002) Good cell culture practice. ECVAM good cell culture practice task force report 1. Altern Lab Anim 30(4):407-414

Hogberg HT, Kinsner-Ovaskainen A, Hartung T, Coecke S, Bal-Price AK (2009) Gene expression as a sensitive endpoint to evaluate cell differentiation and maturation of the developing central nervous system in primary cultures of rat cerebellar granule cells (CGCs) exposed to pesticides. Toxicol Appl Pharmacol 235(3):268-286

Hussman JP, Chung RH, Griswold AJ et al (2011) A noise-reduction GWAS analysis implicates altered regulation of neurite outgrowth and guidance in autism. Mol Autism 2(1):1

Ishido M, Suzuki J (2010) Inhibition by rotenone of mesencephalic neural stem-cell migration in a neurosphere assay in vitro. Toxicol In Vitro 24(2):552-557

Jones LB, Stanwood GD, Reinoso BS et al (2000) In utero cocaineinduced dysfunction of dopamine D1 receptor signaling and abnormal differentiation of cerebral cortical neurons. J Neurosci 20(12):4606-4614

Joshi S, Guleria RS, Pan J et al (2006) Ethanol impairs Rho GTPase signaling and differentiation of cerebellar granule neurons in a rodent model of fetal alcohol syndrome. Cell Mol Life Sci 63(23):2859-2870

Judson R, Kavlock R, Martin M et al (2013) Perspectives on validation of high-throughput assays supporting 21 st century toxicity testing. ALTEX 30(1):51-66

Kadereit S, Zimmer B, van Thriel C, Hengstler JG, Leist M (2012) Compound selection for in vitro modeling of developmental neurotoxicity. Front Biosci 17:2442-2460

Keller H, Zadeh AD, Eggli P (2002) Localised depletion of polymerised actin at the front of Walker carcinosarcoma cells increases the speed of locomotion. Cell Motil Cytoskeleton 53(3):189-202

Kubo T, Yamaguchi A, Iwata N, Yamashita T (2008) The therapeutic effects of Rho-ROCK inhibitors on CNS disorders. Ther Clin Risk Manag 4(3):605-615

Kuegler PB, Zimmer B, Waldmann T et al (2010) Markers of murine embryonic and neural stem cells, neurons and astrocytes: reference points for developmental neurotoxicity testing. ALTEX 27(1):17-42

Lefranc F, Sauvage S, Van Goietsenoven G et al (2009) Narciclasine, a plant growth modulator, activates Rho and stress fibers in glioblastoma cells. Mol Cancer Ther 8(7):1739-1750

Leist M, Efremova L, Karreman C (2010) Food for thought... considerations and guidelines for basic test method descriptions in toxicology. ALTEX 27(4):309-317

Leist M, Hasiwa N, Daneshian M, Hartung T (2012) Validation and quality control of replacement alternatives - current status and future challenges. Toxicol Res 1:8-22

LoPachin RM, Ross JF, Reid ML, Das S, Mansukhani S, Lehning EJ (2002) Neurological evaluation of toxic axonopathies in rats: acrylamide and 2,5-hexanedione. Neurotoxicology 23(1):95-110

Lotharius J, Falsig J, van Beek J et al (2005) Progressive degeneration of human mesencephalic neuron-derived cells triggered by dopamine-dependent oxidative stress is dependent on the mixedlineage kinase pathway. J Neurosci 25(27):6329-6342

McCormack AL, Thiruchelvam M, Manning-Bog AB et al (2002) Environmental risk factors and Parkinson's disease: selective degeneration of nigral dopaminergic neurons caused by the herbicide paraquat. Neurobiol Dis 10(2):119-127
McEwen BS (1999) Stress and hippocampal plasticity. Annu Rev Neurosci 22:105-122

Mitchell PJ, Hanson JC, Quets-Nguyen AT, Bergeron M, Smith RC (2007) A quantitative method for analysis of in vitro neurite outgrowth. J Neurosci Methods 164(2):350-362

Narro ML, Yang F, Kraft R, Wenk C, Efrat A, Restifo LL (2007) NeuronMetrics: software for semi-automated processing of cultured neuron images. Brain Res 1138:57-75

Niggli V (2003) Microtubule-disruption-induced and chemotacticpeptide-induced migration of human neutrophils: implications for differential sets of signalling pathways. J Cell Sci 116(Pt 5):813-822

Nikolic M (2002) The role of Rho GTPases and associated kinases in regulating neurite outgrowth. Int $\mathrm{J}$ Biochem Cell Biol 34(7):731-745

Poltl D, Schildknecht S, Karreman C, Leist M (2012) Uncoupling of ATP-depletion and cell death in human dopaminergic neurons. Neurotoxicology 33(4):769-779

Quasthoff S, Hartung HP (2002) Chemotherapy-induced peripheral neuropathy. J Neurol 249(1):9-17

Radio NM, Mundy WR (2008) Developmental neurotoxicity testing in vitro: models for assessing chemical effects on neurite outgrowth. Neurotoxicology 29(3):361-376

Radio NM, Breier JM, Shafer TJ, Mundy WR (2008) Assessment of chemical effects on neurite outgrowth in PC12 cells using high content screening. Toxicol Sci 105(1):106-118

Ramm P, Alexandrov Y, Cholewinski A, Cybuch Y, Nadon R, Soltys BJ (2003) Automated screening of neurite outgrowth. J Biomol Screen 8(1):7-18

Ren Y, Liu W, Jiang H, Jiang Q, Feng J (2005) Selective vulnerability of dopaminergic neurons to microtubule depolymerization. J Biol Chem 280(40):34105-34112

Sai Y, Wu Q, Le W, Ye F, Li Y, Dong Z (2008) Rotenone-induced PC12 cell toxicity is caused by oxidative stress resulting from altered dopamine metabolism. Toxicol In Vitro 22(6):1461-1468

Sanchez M, Gastaldi L, Remedi M, Caceres A, Landa C (2007) Rotenone-induced toxicity is mediated by Rho-GTPases in hippocampal neurons. Toxicolog Sci 104(2):352-361

Schierle GS, Hansson O, Leist M, Nicotera P, Widner H, Brundin P (1999) Caspase inhibition reduces apoptosis and increases survival of nigral transplants. Nat Med 5(1):97-100

Schildknecht S, Poltl D, Nagel DM et al (2009) Requirement of a dopaminergic neuronal phenotype for toxicity of low concentrations of 1-methyl-4-phenylpyridinium to human cells. Toxicol Appl Pharmacol 241(1):23-35

Schneider K, Schwarz M, Burkholder I et al (2009) "ToxRTool", a new tool to assess the reliability of toxicological data. Toxicol Lett 189(2):138-144

Schoenenberger F, Krug AK, Leist M, Ferrando-May E, Merhof D (2012) An advanced image processing approach based on parallel growth and overlap handling to quantify neurite growth. Paper presented at the 9th International Workshop on Computational Systems Biology (WCSB), Ulm

Scholz D, Poltl D, Genewsky A, et al. (2011) Rapid, complete and large-scale generation of post-mitotic neurons from the human LUHMES cell line. J Neurochem 19(5):957-971

Slotkin TA, Levin ED, Seidler FJ (2006) Comparative developmental neurotoxicity of organophosphate insecticides: effects on brain development are separable from systemic toxicity. Environ Health Perspect 114(5):746-751

Snow DM, Smith JD, Booze RM, Welch MA, Mactutus CF (2001) Cocaine decreases cell survival and inhibits neurite extension of rat locus coeruleus neurons. Neurotoxicol Teratol 23(3):225-234

Spencer PS, Schaumburg HH, Ludolph AC (2000) Experimental and clinical neurotoxicology, 2nd edn. Oxford University Press, New York 
Stanwood GD, Washington RA, Shumsky JS, Levitt P (2001) Prenatal cocaine exposure produces consistent developmental alterations in dopamine-rich regions of the cerebral cortex. Neuroscience 106(1):5-14

Stephens ML, Andersen M, Becker RA et al (2013) Evidence-based toxicology for the 21st century: opportunities and challenges. ALTEX 30(1):74-104

Stiegler NV, Krug AK, Matt F, Leist M (2011) Assessment of chemical-induced impairment of human neurite outgrowth by multiparametric live cell imaging in high-density cultures. Toxicol Sci 121(1):73-87

Takesono A, Heasman SJ, Wojciak-Stothard B, Garg R, Ridley AJ (2010) Microtubules regulate migratory polarity through Rho/ ROCK signaling in T cells. PLoS ONE 5(1):e8774

van Thriel C, Westerink RH, Beste C, Bale AS, Lein PJ, Leist M (2011) Translating neurobehavioural endpoints of developmental neurotoxicity tests into in vitro assays and readouts. Neurotoxicology 33(4):911-924

Vestergaard-Poulsen P, Wegener G, Hansen B et al (2011) Diffusionweighted MRI and quantitative biophysical modeling of hippocampal neurite loss in chronic stress. PLoS ONE 6(7):e20653

Volbracht C, Leist M, Nicotera P (1999) ATP controls neuronal apoptosis triggered by microtubule breakdown or potassium deprivation. Mol Med 5(7):477-489

Volbracht C, Leist M, Kolb SA, Nicotera P (2001) Apoptosis in caspase-inhibited neurons. Mol Med 7(1):36-48

Volbracht C, van Beek J, Zhu C, Blomgren K, Leist M (2006) Neuroprotective properties of memantine in different in vitro and in vivo models of excitotoxicity. Eur J Neurosci 23(10):2611-2622
Wang D, Lagerstrom R, Sun C, Bishof L, Valotton P, Gotte M (2010) HCA-vision: automated neurite outgrowth analysis. J Biomol Screen 15(9): 1165-1170

Yang D, Kim KH, Phimister A et al (2009) Developmental exposure to polychlorinated biphenyls interferes with experience-dependent dendritic plasticity and ryanodine receptor expression in weanling rats. Environ Health Perspect 117(3):426-435

Yeyeodu ST, Witherspoon SM, Gilyazova N, Ibeanu GC (2010) A rapid, inexpensive high throughput screen method for neurite outgrowth. Curr Chem Genomics 4:74-83

Zhang D, Wang Z, Jin N et al (2001) Microtubule disruption modulates the Rho-kinase pathway in vascular smooth muscle. J Muscle Res Cell Motil 22(2):193-200

Zhang H, Schneider T, Wheeler-Kingshott CA, Alexander DC (2012) NODDI: practical in vivo neurite orientation dispersion and density imaging of the human brain. Neuroimage 61(4):1000-1016

Zikopoulos B, Barbas H (2010) Changes in prefrontal axons may disrupt the network in autism. J Neurosci 30(44):14595-14609

Zimmer B, Kuegler PB, Baudis B et al (2011) Coordinated waves of gene expression during neuronal differentiation of embryonic stem cells as basis for novel approaches to developmental neurotoxicity testing. Cell Death Differ 18(3):383-395

Zimmer B, Lee G, Stiegler NV, et al. (2012) Evaluation of Developmental Toxicants and Signaling Pathways in a Functional Test Based on the Migration of Human Neural Crest Cells. Environ Health Perspect 120(8):1116-1122 